\title{
Fractal supersymmetric QM, geometric probability and the Riemann hypothesis
}

\author{
Carlos Castro ${ }^{1}$, Jorge Mahecha ${ }^{2}$ \\ ${ }^{1}$ Center for Theoretical Studies of Physical Systems, \\ Clark Atlanta University, Atlanta, Georgia, USA \\ ${ }^{2}$ Institute of Physics, University of Antioquia, Medellin, Colombia
}

May 17, 2004

\begin{abstract}
The Riemann's hypothesis (RH) states that the nontrivial zeros of the Riemann zeta-function are of the form $s_{n}=1 / 2+i \lambda_{n}$. Earlier work on the $\mathrm{RH}$ based on supersymmetric QM, whose potential was related to the Gauss-Jacobi theta series, allows to provide the proper framework to construct the well defined algorithm to compute the probability to find a zero (an infinity of zeros) in the critical line. Geometric probability theory furnishes the answer to the difficult question whether the probability that the $\mathrm{RH}$ is true is indeed equal to unity or not. To test the validity of this geometric probabilistic framework to compute the probability if the $\mathrm{RH}$ is true, we apply it directly to the the hyperbolic sine function $\sinh (s)$ case which obeys a trivial analog of the RH (the HSRH). Its zeros are equally spaced in the imaginary axis $s_{n}=0+i n \pi$. The geometric probability to find a zero (and an infinity of zeros) in the imaginary axis is exactly unity. We proceed with a fractal supersymmetric quantum mechanical (SUSY-QM) model implementing the Hilbert-Polya proposal to prove the RH by postulating a Hermitian operator that reproduces all the $\lambda_{n}$ for its spectrum. Quantum inverse scattering methods related to a fractal potential given by a Weierstrass function (continuous but nowhere differentiable) are applied to the fractal analog of the Comtet-BandraukCampbell (CBC) formula in SUSY QM. It requires using suitable fractal derivatives and integrals of irrational order whose parameter $\beta$ is one-half the fractal dimension $(D=1.5)$ of the Weierstrass function. An ordinary SUSY-QM oscillator is also constructed whose eigenvalues are of the form $\lambda_{n}=n \pi$ and which coincide with the imaginary parts of the zeros of the function $\sinh (s)$. Finally, we discuss the relationship to the theory of $1 / f$ noise.
\end{abstract}

\section{Introduction}

Riemann's outstanding hypothesis that the non-trivial complex zeros of the zeta-function $\zeta(s)$ must be of the form $s_{n}=1 / 2 \pm i \lambda_{n}$, is one of most important open problems in pure mathematics. The zeta-function has a relation with the number of prime numbers less than a given quantity and the zeros of zeta are deeply connected with the distribution of primes [1]. References $[2,3,4]$ are devoted to the mathematical properties of the zeta-function.

The RH has also been studied from the point of view of physics (e.g., [5, 6, 7, $8,9]$ ). For example, the spectral properties of the $\lambda_{n}$ 's are associated with the 
random statistical fluctuations of the energy levels (quantum chaos) of a classical chaotic system [8]. Montgomery [10] has shown that the two-level correlation function of the distribution of the $\lambda_{n}$ 's coincides with the expression obtained by Dyson with the help of random matrices corresponding to a Gaussian unitary ensemble. Planat [11] has found a link between RH and the called $1 / f$ noise. Wu and Sprung [12] have numerically shown that the lower lying non-trivial zeros can be related to the eigenvalues of a Hamiltonian having a fractal structure. For a recent and nice discussion on several quantum hamiltonians related to the prime numbers distribution and the zeros of the zeta function see the work by Rosu [13].

Since the literature on the topic is rather extensive we refer the reader to a nice collection of zeta-related papers which can be found in Ref. [14].

Scattering theory on real and $p$-adic symmetric spaces produces $S$-matrices involving the Riemann zeta function [15]. Scattering on the noncompact finite area fundamental domain of $S L(2, Z)$ on the real hyperbolic plane was studied long ago by Fadeev and Pavlov [16], and more recently by Planat and Perrine [17] within the context of the deep arithmetical properties underlying the physics of $1 / f$ noise.

Scattering matrix $s$-wave amplitudes for scattering in the Poincare disk can be expressed in the form [18]:

$$
S=\frac{c(k)}{c(-k)}=\frac{\zeta(i k) \zeta(1-i k)}{\zeta(1+i k) \zeta(-i k)}=e^{i 2 \delta_{0}(k)},
$$

where $c(k)$ are the Harish-Chandra $c$-functions (Jost functions). The Jost functions are defined whether the space is symmetric or not, and whether a suitable potential is introduced or not. One may notice that when $k$ is real-valued the numerator of (1) is the complex conjugate of the denominator and for this reason one can write $S(k)$ as a pure phase factor as indicated in the r.h.s. However when $k$ is complex-valued this is no longer the case and $S(k)$ is no longer given by a pure phase factor. For example, the complex poles of $S(k)$ correspond to the zeros of the zeta functions in the denominator and to their poles in the numerator. $s$-wave scattering by a potential with a cutoff have been recently studied in [19], where the complex zeros of the Jost functions yield the complex poles of the $S$-matrix that are located on a horizontal line (below the real axis) and which can be mapped into the critical line of zeros of the Riemann zeta function. They represent resonances. For example, in the case of $s$-wave scattering in the hyperbolic plane (Poincare disk) one can show that the complex-poles of the $S$-matrix correspond to the nontrivial zeros when,

$$
k_{n}=i\left(1 / 2+i \lambda_{n}\right)
$$

Hence, a Wick rotation of the Riemann critical line yields the complex momenta associated with the double poles of the $S$-matrix above; i.e. the double zeros of the denominator. If one could find a physical reason why the complex double poles of the $S$-matrix should always occur in complex-conjugate pairs:

$$
-i k_{n}=\left(1+i k_{n}\right)^{*}=1-i k_{n}^{*} \Rightarrow k_{n}=i\left(1 / 2+i \lambda_{n}\right),
$$


one would have found a physical proof of the RH. Pigli has discussed why scattering theory on real and $p$-adic systems involving the Riemann zeta function belong to a wide class of integrable models that can be unified into an Adelic integrable systems whose $S$-matrix involves the Dirichlet, Langlands, Shimura, $L$-functions.

In this work we will also invoke an integrability property associated with the quantum inverse scattering problem associated with a (fractal) SUSY QM model that yields the one-to-one correspondence among the imaginary parts of the zeta zeros $\lambda_{n}$ with the phases $\alpha_{n}$ of a fractal Weierstrass function. One could also consider a stochastic process having an underlying hidden Parisi-Sourlas supersymmetry, as the effective motion of a particle in a potential which can be expanded in terms of an infinite collection of $p$-adic harmonic oscillators (See in [20]). But in this case we will focus entirely on a fractal SUSY QM model with a judicious fractal potential.

$\mathrm{Wu}$ and Sprung have made a very insightful and key remark pertaining the conundrum of constructing a one-dimensional integrable and time-reversal quantum Hamiltonian to model the imaginary parts of the zeros of zeta as an eigenvalue problem. This riddle of merging chaos with integrability is solved by choosing a fractal local potential that captures the chaotic dynamics inherent with the zeta zeros.

By a fractal SUSY QM model studied here, we do not mean systems with fractional supersymmetries which are common in the string and $M$-theory literature, but a Hamiltonian operator that admits a factorization into two factors involving fractional derivative operators whose irrational order is one-half of the fractal dimension of the fractal potential. A model of fractal spin has been constructed by Wellington da Cruz [21] in connection to the fractional quantum Hall effect based on the filling factors associated with the Farey fractions. The self-similarity properties of the Farey fractions are widely known to posses remarkable fractal properties [22]. For further details of the validity of the RH based on the Farey fractions and the Franel-Landau [23] shifts we refer to the literature on the zeta function.

In previous work $[20,24,25]$ we have already explored some possible strategies which could lead to a solution of the problem. The last one was based on the relation of the non-trivial zeros of the $\zeta$-function with the orthogonality of eigenfunctions of the appropriately chosen operator (see also [26, 27, 28]). We have not assumed any ad-hoc symmetries like conformal invariance, but in fact, we shown why the $t \rightarrow 1 / t$ symmetry is in direct correlation with the $s^{\prime} \rightarrow 1-s^{\prime}$ symmetry of the Riemann's fundamental identity $Z\left(s^{\prime}\right)=Z\left(1-s^{\prime}\right)$, the function $Z$ is the Riemann fundamental function defined in (12). This was the clue of our proposal to proof the $\mathrm{RH}$.

In this work we illustrate the method in [25] by applying it to the study of the zeros of a very simple function, the $\sinh (s)$. The proof that the zeros of $\sinh (s)$ are given by $0+i y_{n}=0+i n \pi$ is trivial. Nevertheless, one can still furnish another approach following the same steps as the work in [25] about the $\mathrm{RH}$.

The contents of this work are the following. In Section 2 we review our 
previous work on the RH [25] and concentrate in 2.2 on a SUSY QM model whose potential is related to the Gauss-Jacobi theta series. The inner product of the eigenfunctions $\psi_{s}(t), \psi_{s}(1 / t)$ of the partner (non-Hermitian) Hamiltonians $H_{A}, H_{B}$ is given by $Z(a s+b)$ while their spectrum is $s(1-s)$ which happens to be real only in the critical line (location of the nontrivial zeta zeros) and in the real axis (location of the trivial zeta zeros). In 2.3 some important remarks about the Eisenstein series and our approach are made. In Section 3 we present a proof of the HSRH, the hyperbolic sine version of the RH.

In Sections 4 and 4.4 a discussion of a completely different approach to this problem is provided where we construct the well defined algorithm to compute the geometric probability to find a zero (an infinity of zeros) in the critical line; i.e. we show how to find the probability that the $\mathrm{RH}$ is true; when $P=1$ the $\mathrm{RH}$ is true and when $P<1$, it is false. In Section 5 we consider the ordinary SUSY QM model solution to find the imaginary parts of the zeros of the $\sinh (s)$ function, and finally we construct the fractional (fractal) supersymmetric quantum mechanical (SUSY-QM) model whose spectrum yields the imaginary parts $\lambda_{n}$ of the nontrivial zeros of zeta. It is based on a quantum inverse scattering method related to a fractal potential given by a Weierstrass function (continuous but nowhere differentiable) that is present in the fractal analog of the Comtet-Bandrauk-Campbell (CBC) formula in SUSY QM. It requires using suitable fractal derivatives and integrals of irrational order whose parameter $\beta$ is one-half the fractal dimension of the Weierstrass function. In the concluding remarks we show the relation to the theory of $1 / f$ noise.

\section{Nontrivial $\zeta$ 's zeros as an orthogonality rela- tion}

\subsection{The scaling operators related to the Gauss-Jacobi theta series}

Our proposal to compute the geometric probability begins with the introduction of the appropriate generalized scaling operator $D_{1}$

$$
D_{1}=-\frac{d}{d \ln t}+\frac{d V}{d \ln t}+k,
$$

such that its eigenvalues $s$ are complex-valued, and its eigenfunctions are given by

$$
\psi_{s}(t)=t^{-s+k} e^{V(t)} .
$$

$D_{1}$ is not self-adjoint since its eigenvalues are complex valued numbers $s$. We also define the operator dual to $D_{1}$ as follows,

$$
D_{2}=\frac{d}{d \ln t}+\frac{d V}{d \ln t}+k
$$


that is related to $D_{1}$ by the substitution $t \rightarrow 1 / t$ and by noticing that

$$
\frac{d V(1 / t)}{d \ln (1 / t)}=-\frac{d V(1 / t)}{d \ln t},
$$

where $V(1 / t)$ is not equal to $V(t)$.

Since $V(t)$ can be chosen arbitrarily, we choose it to be related to the Bernoulli string spectral counting function, given by the Jacobi theta series,

$$
e^{2 V(t)}=\sum_{n=-\infty}^{\infty} e^{-\pi n^{2} t^{l}}=2 \omega\left(t^{l}\right)+1 .
$$

This choice is justified in part by the fact that Jacobi's theta series $\omega$ has a deep connection to the integral representations of the Riemann zeta-function [29].

Latter arguments will rely also on the following related function defined by Gauss,

$$
G(1 / x)=\sum_{n=-\infty}^{\infty} e^{-\pi n^{2} / x}=2 \omega(1 / x)+1
$$

where $\omega(x)=\sum_{n=1}^{\infty} e^{-\pi n^{2} x}$. Then, our $V$ is such that $e^{2 V(t)}=G\left(t^{l}\right)$. We defined $x$ as $t^{l}$. We call $G(x)$ the Gauss-Jacobi theta series (GJ).

Thus we have to consider a family of $D_{1}$ operators, each characterized by two real numbers $k$ and $l$ which can be chosen arbitrarily. The measure of integration $d \ln t$ is scale invariant. Let us mention that $D_{1}$ is also invariant under scale transformations of $t$ and $F=e^{V}$ since $d V /(d \ln t)=d \ln F /(d \ln t)$. In [26] only one operator $D_{1}$ is introduced with the number $k=0$ and a different (from ours) definition of $F$.

We define the inner product as follows,

$$
\langle f \mid g\rangle=\int_{0}^{\infty} f^{*} g \frac{d t}{t}
$$

Based on this definition, the inner product of two eigenfunctions of $D_{1}$ is

$$
\begin{aligned}
\left\langle\psi_{s_{1}} \mid \psi_{s_{2}}\right\rangle & =\alpha \int_{0}^{\infty} e^{2 V} t^{-s_{12}+2 k-1} d t \\
& =\frac{2 \alpha}{l} Z\left[\frac{2}{l}\left(2 k-s_{12}\right)\right],
\end{aligned}
$$

where we have denoted $s_{12}=s_{1}^{*}+s_{2}=x_{1}+x_{2}+i\left(y_{2}-y_{1}\right)$ and used the expressions (7) and (12).

We notice that $\left\langle\psi_{s_{1}} \mid \psi_{s_{2}}\right\rangle=\left\langle\psi_{s_{o}} \mid \psi_{s}\right\rangle$. Thus, the inner product of $\psi_{s_{1}}$ and $\psi_{s_{2}}$ is equivalent to the inner product of $\psi_{s_{o}}$ and $\psi_{s}$, where $s_{o}=1 / 2+i 0$ and $s=s_{12}-1 / 2$. Constant $\alpha$ is to be appropriately chosen so that the inner product 
in the critical domain is semi-positively definite. The integral is evaluated by introducing a change of variables $t^{l}=x$ (which gives $\left.d t / t=(1 / l) d x / x\right)$ and using the result provided by the equation (8), given in Karatsuba and Voronin's book [2]. Function $Z$ in (12) can be expressed in terms of the Jacobi theta series, $\omega(x)$ defined by $(7)$ (see $[3])$,

$$
\begin{aligned}
\int_{0}^{\infty} \sum_{n=1}^{\infty} e^{-\pi n^{2} x} x^{s / 2-1} d x & = \\
& =\int_{0}^{\infty} x^{s / 2-1} \omega(x) d x \\
& =\frac{1}{s(s-1)}+\int_{1}^{\infty}\left[x^{s / 2-1}+x^{(1-s) / 2-1}\right] \omega(x) d x \\
& =Z(s)=Z(1-s),
\end{aligned}
$$

where

$$
Z(s) \equiv \pi^{-s / 2} \Gamma\left(\frac{s}{2}\right) \zeta(s)
$$

which obeys the functional relation $Z(s)=Z(1-s)$.

Since the right-hand side of $(11)$ is defined for all $s$, this expression gives the analytic continuation of the function $Z(s)$ to the entire complex $s$-plane [3]. In this sense the fourth "=" in (11) is not a genuine equality. Such an analytic continuation transforms this expression into the inner product, defined by (10).

A recently published report by Elizalde, Moretti and Zerbini [28] (containing comments about the first version of our paper [30]) considers in detail the consequences of the analytic continuation implied by equation (11). One of the consequences is that equation (10) loses the meaning of being a scalar product. Arguments by Elizalde et al. [28] show that the construction of a genuine inner product is impossible.

Therefore from now on we will loosely speak of a "scalar product" realizing that we do not have a scalar product as such. The crucial problem is whether there are zeros outside the critical line (but still inside the critical strip) and not the interpretation of equation (10) as a genuine inner product. Despite this, we still rather loosely refer to this mapping as a scalar product. The states still have a real norm squared, which however need not to be positive-definite.

Here we must emphasize that our arguments do not rely on the validity of the zeta-function regularization procedure [31], which precludes a rigorous interpretation of the right hand side of (11) as a scalar product. Instead, we can simply replace the expression "scalar product of $\psi_{s_{1}}$ and $\psi_{s_{2}}$ " by the map $S$ of complex numbers defined as

$$
\begin{aligned}
& S: \quad \mathcal{C} \otimes \mathcal{C} \rightarrow \mathcal{C} \\
& \left(s_{1}, s_{2}\right) \mapsto S\left(s_{1}, s_{2}\right)=-Z(a s+b),
\end{aligned}
$$


where $s=s_{1}^{*}+s_{2}-1 / 2$ and $a=-2 / l ; b=(4 k-1) / l$. In other words, our arguments do not rely on an evaluation of the integral $\left\langle\psi_{s_{1}} \mid \psi_{s_{2}}\right\rangle$, but only on the mapping $S\left(s_{1}, s_{2}\right)$, defined as the finite part of the integral (10). The kernel of the map $S\left(s_{1}, s_{2}\right)=-Z(a s+b)$ is given by the values of $s$ such that $Z(a s+b)=0$, where $\left\langle\psi_{s_{1}} \mid \psi_{s_{2}}\right\rangle=\left\langle\psi_{s_{o}} \mid \psi_{s}\right\rangle$ and $s_{o}=1 / 2+i 0$. Notice that $2 b+a=4(2 k-1) / l$. We only need to study the "orthogonality" (and symmetry) conditions with respect to the "vacuum" state $s_{O}$ to prove the RH. By symmetries of the "orthogonal" states to the "vacuum" we mean always the symmetries of the kernel of the $S$ map.

The "inner" products are trivially divergent due to the contribution of the $n=0$ term of the GJ theta series in the integral (10). From now on, we denote for "inner" product in (10) and (13) as the finite part of the integrals by simply removing the trivial infinity. We shall see in the next paragraphs, that this "additive" regularization is in fact compatible with the symmetries of the problem.

We can easily show that if $a$ and $b$ are such that $2 b+a=1$, then the symmetries of all the states $\psi_{s}$ orthogonal to the "vacuum" state are preserved by any map $S$, equation (13), which leads to $Z(a s+b)$. In fact, if the state associated with the complex number $s=x+i y$ is orthogonal to the "vacuum" state and the "scalar product" is given by $Z(a s+b)=Z\left(s^{\prime}\right)$, then the Riemann zeta-function has zeros at $s^{\prime}=x^{\prime}+i y^{\prime}, s^{*}, 1-s^{\prime}$ and $1-s^{* *}$. If we equate $a s+b=s^{\prime}$, then $a s^{*}+b=s^{*}$. Now, $1-s^{\prime}$ will be equal to $a(1-s)+b$, and $1-s^{*}$ will be equal to $a\left(1-s^{*}\right)+b$, if, and only if, $2 b+a=1$. Therefore, all the states $\psi_{s}$ orthogonal to the "vacuum" state, parameterized by the complex number $1 / 2+i 0$, will then have the same symmetry properties with respect to the critical line as the nontrivial zeros of zeta. See Figure 2.

Notice that our choice of $a=-2 / l$ and $b=(4 k-1) / l$ is compatible with this symmetry if $k$ and $l$ are related by $l=4(2 k-1)$. Conversely, if we assume that the orthogonal states to the "vacuum" state have the same symmetries of $Z(s)$, then $a$ and $b$ must be constrained to obey $2 b+a=1$. It is clear that a map with arbitrary values of $a$ and $b$ does not preserve the above symmetries and for this reason we have now that $s^{\prime}=a s+b=a(s-1 / 2)+1 / 2$

Therefore, concluding, the inner product $\left\langle\psi_{s_{1}} \mid \psi_{s_{2}}\right\rangle$ is equal to $\left\langle\psi_{s_{o}} \mid \psi_{s}\right\rangle=$ $Z[a(s-1 / 2)+1 / 2]=Z\left(s^{\prime}\right)$ where $s=s_{1}^{*}+s_{2}-1 / 2$. For example, if we set $l=-2$, then $k=1 / 4, a=1, b=0$, and consequently $s^{\prime}=s$ in this case; i.e. the position of the zeros $s$ coincide with the location of the orthogonal states $\psi_{s}$ to the reference state $\psi_{s_{o}}$. A figure displaying this particular case is Figure 3.

\subsection{The zeros from supersymmetric quantum mechanics}

A different way to prove the RH can be found following the Hilbert-Polya proposal by finding Hermitian operators that reproduce the zeros as its spectrum. We will see also that this symmetry of the "vacuum", is also compatible with 
the isospectral property of the two partner Hamiltonians,

$$
H_{A}=D_{2} D_{1}=\left[\frac{d}{d \ln t}-\frac{d V(1 / t)}{d \ln (1 / t)}+k\right]\left[-\frac{d}{d \ln t}+\frac{d V(t)}{d \ln t}+k\right],
$$

and

$$
H_{B}=D_{1} D_{2}=\left[-\frac{d}{d \ln t}+\frac{d V(t)}{d \ln t}+k\right]\left[\frac{d}{d \ln t}-\frac{d V(1 / t)}{d \ln (1 / t)}+k\right] .
$$

Notice that $V(1 / t) \neq V(t)$ and for this reason $D_{2}$ is not the "adjoint" of $D_{1}$. Operators defined on the half line do not admit an adjoint extension, in general. Hence, the partner Hamiltonians $H_{A}, H_{B}$ are not (self-adjoint) Hermitian operators like it occurs in the construction of SUSY QM. Consequently their eigenvalues are not real in general.

Nevertheless one can show by inspection that if, and only if, $\psi_{s}(1 / t)=$ $\psi_{1-s}(t)$ then both partner Hamiltonians are isospectral (like in SUSY QM) whose spectrum is given by $s(1-s)$ and the corresponding eigenfunctions are,

$$
H_{A} \psi_{s}(t)=s(1-s) \psi_{s}(t), \quad H_{B} \psi_{s}(1 / t)=s(1-s) \psi_{s}(1 / t)
$$

Firstly by a direct evaluation one can verify,

$$
D_{1} \psi_{s}(t)=s \psi_{s}(t), D_{2} \psi_{s}(1 / t)=s \psi_{s}(1 / t)
$$

i.e. $\psi_{s}(t)$ and $\psi_{s}(1 / t)$ are eigenfunctions of the $D_{1}$ and $D_{2}$ operators respectively with complex eigenvalue $s$. Secondly, if, and only if, the condition $\psi_{s}(1 / t)=$ $\psi_{1-s}(t)$ is satisfied, then it follows that:

$$
\begin{aligned}
& H_{B} \psi_{s}(1 / t)=D_{1} D_{2} \psi_{s}(1 / t)=s D_{1} \psi_{s}(1 / t)= \\
& s D_{1} \psi_{1-s}(t)=s(1-s) \psi_{1-s}(t)=s(1-s) \psi_{s}(1 / t) \text {, }
\end{aligned}
$$

meaning that $\psi_{s}(1 / t)$ is an eigenfunction of $H_{B}$ with $s(1-s)$ eigenvalue, and:

$$
\begin{aligned}
& H_{A} \psi_{s}(t)=D_{2} D_{1} \psi_{s}(t)=s D_{2} \psi_{s}(t)= \\
& s D_{2} \psi_{1-s}(1 / t)=s(1-s) \psi_{1-s}(1 / t)=s(1-s) \psi_{s}(t) \text {, }
\end{aligned}
$$

meaning that $\psi_{s}(t)$ is an eigenfunction of $H_{A}$ with $s(1-s)$ eigenvalue.

Therefore, under condition $\psi_{s}(1 / t)=\psi_{1-s}(t)$ the non-Hermitian partner Hamiltonians are isospectral. The spectrum is $s(1-s)$. The operators $H_{A}$ and $H_{B}$ are quadratic in derivatives like the Laplace-Beltrami operator and involve two generalized dilatation operators $D_{1}$ and $D_{2}$. Notice the most important results of this section:

1. On the critical Riemann line, because $\mathcal{R} e(s)=1 / 2 \rightarrow 1-s=s^{*}$, the eigenvalues are real since $s(1-s)=s s^{*}$ is real. The function $Z(s)$ is also real on the critical line as a result of the Schwarz reflection principle of the function $Z(s)=u(s)+i v(s)$ obeying $u\left(s^{*}\right)=u(s) ; v\left(s^{*}\right)=-v(s)$ so due to the condition $1-s=s^{*}$ (true only for points living in the critical line) and 
$Z(s)=Z(1-s)=Z\left(s^{*}\right)=Z^{*}(s)$, allows to infer that $Z(s)$ is real-valued in the critical line.

2. On the real line, the eigenvalues $s(1-s)$ are trivially real.

Therefore, the spectrum $s(1-s)$ of the two partner (non-Hermitian) Hamiltonians is real-valued when $s$ falls in the critical line (location of nontrivial zeros) and when $s$ falls in the real line (location of trivial zeros). Hence, the SUSY QM model yields the precise location of the lines of the trivial and nontrivial zeros of zeta!. Notice the similarity of these results with the eigenvalues of the Laplace Beltrami operator in the hyperbolic plane associated with the chaotic billiard living on a surface of constant negative curvature. In that case the Selberg zeta function (which obeys the $\mathrm{RH}$ ) played a crucial role [6].

The states $\psi_{s}(t)$ constitute an over-complete basis. An orthonormal discrete and complete basis can be found, when $s_{n}=1 / 2+i \lambda_{n}$, by simply recurring to the orthogonality conditions of the states $\psi_{s_{n}}$ with respect to the "ground" or "vacuum" state $\psi_{s_{o}}=\psi_{1 / 2+i 0}$. By starting with $\psi_{0}(t)=\psi_{s_{o}}$ the first orthonormal state is $\psi_{1}(t)=a_{11} \psi_{s_{1}}$. The naive normalization condition $\left\langle\psi_{s_{1}} \mid \psi_{s_{1}}\right\rangle=a_{11}^{2} Z(1 / 2+i 0)=1$ would have yielded the value of the coefficient $a_{11}$. However, there is a problem because the value $Z(1 / 2+i 0)=-3.97697<0$. The function $Z(1 / 2+i y)$ is real for all values of $y$ and since there are an infinity of zeros in the critical line these real values are not always positive definite. There are oscillations between positive and negative values. For this reason, the correct way to define the normalization condition (and "inner" products) is to take the absolute values: $\left|\left\langle\psi_{s_{1}} \mid \psi_{s_{1}}\right\rangle\right|=a_{11}^{2}|Z(1 / 2+i 0)|=1$, and $a_{11}$ can be derived satisfactory.

Iterating this procedure gives:

$$
\psi_{n}(t)=\sum_{m=1}^{n} a_{n m} \psi_{s_{m}}(t),
$$

for all $s_{m}=1 / 2+i \lambda_{m}$ such that $m=1,2, \ldots n$. The coefficients $a_{m n}$ are determined by imposing the orthogonality and normalization conditions with the provision that absolute values have to be taken:

$$
\left|\left\langle\psi_{m^{\prime}}(t) \mid \psi_{m}(t)\right\rangle\right|=\delta_{m^{\prime} m} .
$$

In this fashion the discrete and complete orthonormal basis $\psi_{1}(t), \psi_{2}(t), \ldots$ $\psi_{n}(t), \psi_{n+1}(t)$, all the way to $n=\infty$ of states is constructed in terms of the eigenfunctions $\psi_{s}(t), \psi_{s}(1 / t)$ of the two partner $H_{A}, H_{B}$ Hamiltonians associated with a SUSY QM model and which is entirely based on the locations of the nontrivial zeros of zeta in the critical line.

To sum up, the inversion properties under $t \rightarrow 1 / t$ of the eigenfunctions of the infinite family of differential operators, $D_{1}^{(k, l)}(t)$ and $D_{2}^{(k, l)}(1 / t)$, compatible with the existence of an invariant "vacuum", are responsible for the isospectral condition of the partner non-Hermitian Hamiltonians, $H_{A}$ and $H_{B}$, like it occurs in SUSY QM. The spectrum $s(1-s)$ is real in the critical line (location of the nontrivial zeros) and in the real line (location of the trivial zeros). The quantum 
inverse scattering problem associated with a fractal SUSY QM model which yields the imaginary parts of the nontrivial zeros consistent with the HilbertPolya proposal to prove the RH will be studied in Section 5. The supersymmetric ground state is precisely that associated with $s_{o}=1 / 2+i 0$. Rosu has recast our SUSY QM wave equations into a transparent SUSY QM form [13].

\subsection{A remark on Eisenstein series}

Let's emphazise the importance of the Eisenstein series $E(s, z)$ being the twodimensional analog of what we did in Section 2.2.

Using the fundamental function $Z(s)=Z(1-s)$ one constructs the function $I(s, z)$ defined as $I(s, z)=Z(2 s) E(s, z)$ which obeys the same functional relation as the $Z(s)$ (See [32]). Notice the crucial $2 s$ argument inside the $Z$. It reads:

$$
I(s, z)=I(1-s, z) .
$$

Note that it is the function $I(s, z)$ and not the $E(s, z)$ that obeys the same functional relation as $Z(s)$.

The function $I(s, z)$ admits also a theta series representation, and the eigenfunctions of the 2-D Laplacian in the hyperbolic plane are given by the $E(s, z)$. The eigenvalue problem for the two-dimensional Laplacian in the hyperbolic plane is:

$$
y^{2}\left(\frac{\partial^{2}}{\partial x^{2}}+\frac{\partial^{2}}{\partial y^{2}}\right) E(s, z)=-s(1-s) E(s, z),
$$

where $z=x+i y$ (notice the eigenvalues). One has used the Laplace-Beltrami differential operator in non-Euclidean geometries. The hyperbolic metric is conformally flat and for this reason the hyperbolic Laplacian must be conformal to the ordinary Laplacian in flat spaces. This explains the prefactor of $y^{2}$ in front of the ordinary Laplacian.

Since the Laplacian is two-dimensional, this means that the Eisenstein series $E(s, z)$ are the 2-D version ( $s, z$ are both complex and independent) of our eigenfunctions $\psi(s, t)$ of the 1-D Laplacian-like operator obeying:

$$
H_{A} \psi(s, t)=s(1-s) \psi(s, t)
$$

and

$$
H_{B} \psi(s, 1 / t)=s(1-s) \psi(s, 1 / t) .
$$

The $H_{A}, H_{B}$ are the two partner Hamiltonians in our SUSY-QM model, which is a 1-D model defined on half of the real line: $0<t<\infty$.

Whereas the hyperbolic plane where the 2-D Laplacian acts, is represented as the upper half of the complex plane given by the coordinates $z$.

Concluding, the " $t$ " in our $\psi(s, t)$ does correspond to the " $z$ " in $E(s, z)$. Of course, on the Riemann critical line the spectrum $s(1-s)$ is real (and on the real line, trivial zeros). The advantage in our approach is that the inner products of our eigenfunctions $\psi(s, t)$ yield the fundamental function $Z(a s+b)$ and there is a one-to-one correspondence between the zeta zeros and the ortogonality conditions on the $\psi(s, t)$ eigenfunctions. 


\section{The analog of the Riemann hypothesis for the function $\sinh (\mathbf{z})$}

It can be proved in an straightforward way that the function of complex variable $\sinh (z)$ has its zeros in the imaginary axis where the real parts of all the zeros are zero $s=0+i \pi n$ by simply using the addition law of the $\operatorname{sines} \sinh (z)=$ $-i \sin (i z)=-i \sin (i x-y)=\sinh x \cos y+i \cosh x \sin y=0 \Rightarrow x=0, y=\pi n$.

We note that $z=0+i 0$ is a trivial zero. In this section we will propose a different strategy, based on a representation of this function in the form of a scalar product of two functions. And in the next section, we will compute the probability to find a zero (an infinity of zeros) in the imaginary axis and prove that it is exactly given by unity.

Our proposal begins with introducing the appropriate operator $D_{1}$ which is not self adjoint,

$$
D_{1}=\frac{d}{d \chi}
$$

such that its eigenvalues $s$ are complex-valued, and its eigenfunctions are given by

$$
\psi_{s}(\chi)=e^{s \chi}
$$

We restrict $\chi$ to be into the interval $[-1,1]$.

We will only suppose that the following symmetries of our test function $\sinh (z)$ are known,

$$
\sinh (z+2 i \pi n)=\sinh (z), \sinh (-z)=-\sinh (z),
$$

$n$ is an integer. Note that $\sinh (z)$ obeys the Schwarz reflection condition $\sinh ^{*}(z)=\sinh \left(z^{*}\right)$. Therefore if $\sinh (s)=0$, then $\sinh ^{*}(s)=\sinh \left(s^{*}\right)=0$, which means that $s^{*}$ is also a zero if $s$ is a zero. Thus the zeros appear in pairs of complex conjugates. Since $\sinh (-s)=-\sinh (s)$, the zeros are also reflection symmetric with respect to the origin, hence there is a quartet of zeros, living in the vertices of a rectangle, symmetrically distributed with respect to the horizontal and vertical axis. Like it occurs with the zeta zeros, one has a rectangle whose vertices are symmetrical located with respect to the Riemann critical line and horizontal axis (See Figure 2).

We can simply show why the function of the complex variable $z$ (See Figure $1)$,

$$
G(z)=\frac{\sinh (z)}{z}=G(-z),
$$

that has analogous symmetries as the $Z(s)$ in the Riemann zeta case (12), can be obtained from an "inner" product as follows:

$$
\langle f \mid g\rangle=\int_{-1}^{1} f^{*} g d \chi .
$$


The reason we choose the lower and upper limits $[-1,1]$ is because we have to introduce a cutoff to regularize the "inner" products.

Based on this definition, the "inner" product of two eigenfunctions of $D_{1}$ is indeed given by,

$$
\left\langle\psi_{s_{1}} \mid \psi_{s_{2}}\right\rangle=\int_{-1}^{1} e^{\left(s_{1}^{*}+s_{2}\right) \chi} d \chi=\frac{\sinh \left(s_{1}^{*}+s_{2}\right)}{s_{1}^{*}+s_{2}}=G(s)
$$

where $s=s_{1}^{*}+s_{2}$. Thus, $\left\langle\psi_{s_{1}} \mid \psi_{s_{2}}\right\rangle=G\left(s_{1}^{*}+s_{2}\right)=G(s)$.

The (known) zeros of the function $G(s)$ are given by the kernel of the map from $C^{2} \rightarrow C$ defined as $S\left(s_{1}, s_{2}\right)=G(s)=\sinh (s) / s$ where $s=s_{1}^{*}+s_{2}$. It is very important to emphasize that whether we have rigorously speaking a true inner product or not is irrelevant to the calculation of the geometric probability (in the next section) because we will be just "counting" the number of possible pairings $\left(s_{1}, s_{2}\right)$ that generate a given zero $s$ to evaluate the probabilities. Hence, the inner product property is not essential. It is the fact that we are searching for the pairings that generate the kernel of the map $S\left(s_{1}, s_{2}\right)=G(s)=G\left(s_{1}^{*}+s_{2}\right)$.

Concluding, the "scalar" product of $\psi_{s_{1}}$ and $\psi_{s_{2}}$ defines the following map $S$ :

$$
\begin{aligned}
S: \mathcal{C} \otimes \mathcal{C} & \rightarrow \mathcal{C} \\
\left(s_{1}, s_{2}\right) & \mapsto S\left(s_{1}, s_{2}\right)=G\left(s_{1}^{*}+s_{2}\right)=G(s)=\frac{\sinh (s)}{s} .
\end{aligned}
$$

The kernel of the map $S\left(s_{1}, s_{2}\right)=G(s)$ is by definition given by the values of $s$ such that $G(s)=0$; i.e. by the zeros.

In the imaginary axis $s=0+i y$, we have that $s^{*}=-s$, and due to general reflection symmetry of the $G(s)$ function with respect to the origin $0+i 0$ we have: $G(s)=G(-s)=G\left(s^{*}\right)=G^{*}(s)$ (Schwarz reflection principle) and hence $G(s)$ is real-valued in the imaginary axis like the function $Z(s)$ was real valued in the critical line. Thus the function $G(s)$ shares similar symmetries and behaviour as the $Z(s)$ function which reinforces our arguments in favour of a probabilistic appproach to prove the $\mathrm{RH}$.

In the next section we will show in detail how to compute the probabilty to find a zero (an infinity of zeros) in the imaginary axis $Y$ of the $\sinh (s)$ function and we shall find that it is exactly 1 . This is a test that the probabilistic approach to solve the RH is valid. The important point is that both zeros of the $Z(s)$ and $G(s)$ functions can be treated within the framework of geometric probability. A method to locate the $y$ values of the zeros of $\sinh s$ and $\zeta(s)$ using the SUSY QM model is presented in the next sections. Of course one can trivially determine the zeros of $\sinh (s)$, by simply using the sum rules for trigonometric/hyperbolic functions that leads to $s_{n}=0+n \pi$, but we wish to show now how they can also be determined via a SUSY QM model, in addition to the probabilistic method. 


\section{The probabilistic interpretation of the $\mathrm{RH}$}

\subsection{Geometric probability}

Our procedure differs completely from the well known probability proposal of the RH made by Denjoy [33] long ago. In this section we will evaluate the probability to generate a zeta zero resuting from the inner product of pairs of states associated with two complex numbers $\left\langle\psi_{s_{1}} \mid \psi_{s_{2}}\right\rangle=\left\langle\psi_{s_{o}} \mid \psi_{s}\right\rangle=Z(s)=$ 0 . Related to this problem is the celebrated Montgomery-Dyson correlation distribution law [10]:

$$
P=1-\frac{\sin ^{2}(\pi x)}{(\pi x)^{2}}
$$

for the differences among pairs of the imaginary parts of the zeta zeros $\lambda_{i}-\lambda_{j}$ found by Montgomery, assuming the $\mathrm{RH}$ is true, which turned out to agree with the same correlation distribution law for the pairs of eigenvalues of large random hermitean matrices of the Gaussian unitary ensemble (GUE) found by Dyson.

Since this approach based on random matrix theory (RMT) is based on the assumption that the $\mathrm{RH}$ is true, we shall evaluate the probabilities to generate a zeta zero using techniques borrowed from geometric probability theory; i.e. by evaluating probabilities in terms of the ratios of areas and whose results, in turn, will bring us closely to the Montgomery-Dyson probability distribution associated with random matrix models.

Given an orthogonality relation among any two states, we have shown in Section 2 that it can be recast in terms of an orthogonality condition with respect to the vacuum reference state as:

$$
\left\langle\psi_{s_{1}} \mid \psi_{s_{2}}\right\rangle=\left\langle\psi_{s_{o}} \mid \psi_{s}\right\rangle=Z(a s+b)=Z[a(s-1 / 2)+1 / 2]=Z\left(s^{\prime}\right)=0,
$$

where $s \equiv s_{1}^{*}+s_{2}-1 / 2$ and $s_{o}=1 / 2+i 0$. For simplicity we shall fix the value of $a=1$, once and for all, such that the location of the zeta zeros $s^{\prime}$ coincides with $s=s^{\prime}$, the orthogonal state to the vacuum/reference state.

Rigorously speaking one does not have an inner produt as such, however, this does not affect the probability outcome because we will be counting in how many ways the pairs of complex numbers $\left(s_{1}, s_{2}\right)$ generate a zero $s$ from the mapping of $C^{2} \rightarrow C$ defined as $S\left(s_{1}, s_{2}\right)=Z(s)$. Whether the inner product notation $\left\langle\psi_{s_{1}} \mid \psi_{s_{2}}\right\rangle$ is the correct one to use is irrelevant. The relevant issue is that the nontrivial zeros are given by the kernel of the map $S\left(s_{1}, s_{2}\right)=Z(s)=0$.

To be able to solve this problem of computing the probability to find an infinite number of nontrival zeta zeros in the critical line, and wether or not this probability is truly equal to 1 , it will help us to prove that the probability that the infinite number of zeros of the function $\sinh (s)$ fall in the imaginary axis is exactly 1 . All the zeros of the $\sinh (s)$ function fall in the imaginary axis, which is an analogous example of the Riemann zeta zeros. It is straightforward to show why the zeros are $s_{n}=0+i n \pi$. The fact that we can also prove that the probability to find all the zeros of $\sinh (s)$ in the vertical axis is unity is a very good test indeed that our procedure to solve the $\mathrm{RH}$ is valid. 
The way to tackle this problem of computing probabilities to find all the nontrivial zeros is to recast it in terms of geometric probability (stochastic geometry), in particular, what is called the triangle-picking problem inside a given domain. Since one is searching for those configurations of 3 points $s_{1}, s_{2}, s$ of the complex plane in the shape of triangles and lines (when the case the 3 points are colinear) such that the inner products of the states $\left\langle\psi_{s_{1}} \mid \psi_{s_{2}}\right\rangle=\left\langle\psi_{s_{o}} \mid \psi_{s}\right\rangle=Z(s)$ is zero. See Figure 3.

The idea is to "count" in how many ways the pairs $\left(s_{1}, s_{2}\right)$ of complex numbers generate a given zero $s$ and divide by the total number of possible paircombinations to obtain a probability. Geometric probability provides us with the answer!. To every given zero $s$ the average area of all the possible triangles generated from the 3 points $s_{1}, s_{2}, s$ inside a given region in the complex plane is:

$$
\langle A\rangle=\frac{\int A(\text { triangle }) d x_{1} d x_{2} d x d y_{1} d y_{2} d y}{\int d x_{1} d x_{2} d x d y_{1} d y_{2} d y},
$$

where the area of the triangle $A$ sustained by 3 points is given by $1 / 2$ times the absolute value of the determinant of the $3 \times 3$ matrix whose entries are $\left\{\left(x_{1}, x_{2}, x\right) ;\left(y_{1}, y_{2}, y\right) ;(1,1,1)\right\}$ and the integrals can be restricted to the square domain of sides equal to $L$ such that it encloses the zero $s$. Explicitly, the determinant is given by $-x_{1} y+x_{2} y+x y_{1}-x_{2} y_{1}-x y_{2}+x_{1} y_{2}$.

In the triangle-picking case, to compute the probability $P\left(\alpha_{n}+i \beta_{n}\right)$ of generating a zero, $\alpha_{n}+i \beta_{n}$, where $n$ labels the rectangle located in the complex plane whose four vertices:

$$
\alpha_{n}+i \beta_{n} ; \alpha_{n}-i \beta_{n} ; 1-\alpha_{n}-i \beta_{n} ; 1-\alpha_{n}+i \beta_{n},
$$

are the location of possible zeta zeros, we must integrate with respect to the point coordinates inside the entries of the matrix determinant and which must be constrained to obey the following conditions imposed by the inner products:

$$
x=x_{1}+x_{2}-1 / 2=\alpha_{n} \Rightarrow x_{2}=\left(\alpha_{n}+1 / 2\right)-x_{1},
$$

and

$$
y=y_{2}-y_{1}=\beta_{n} \Rightarrow y_{2}=y_{1}+\beta_{n},
$$

leading to a reduced integral with respect the variables $\left(x_{1}, y_{1}\right)$ only as follows:

$$
\left\langle A\left(\alpha_{n}+i \beta_{n}\right)\right\rangle=\frac{\int A(\text { triangle }) d x_{1} d y_{1}}{\int d x_{1} d y_{1}} .
$$

This reduced integral is an explicit function of $\alpha_{n}, \beta_{n}$ that appears in the determinant. Since we face the problem that the complex domain has infinite area we restrict the integrals to a square of size $2 L$, centered in the center of symmetry $1 / 2+i 0$, and enclosing the zero $\alpha_{n}+i \beta_{n}$.

The determinant of the 3 matrix yields,

$$
x_{1}\left(2 y_{1}-\beta_{n}\right)-\left(\alpha_{n}+1 / 2\right) y_{1}+\frac{\beta_{n}}{2} .
$$


After performing the double integral of the determinant with respect to the $x_{1}, y_{1}$ variables, one is left with the value of the average area, after multiplying the determinant by a factor of $1 / 2$. Thus the average area of all the family of triangles associated with each particular zero of the form $\alpha_{n}+i \beta_{n}$ is written $\left\langle A\left(\alpha_{n}, \beta_{n}\right)\right\rangle$.

In the particular case of the Riemann zeros living in the critical line we have that $\alpha_{n}=1 / 2$ and $\beta_{n}=\lambda_{n}$. The areas in the integrand are given in this case by:

$$
\frac{1}{2}\left|x_{1}\left(2 y_{1}-\lambda_{n}\right)-y_{1}+\frac{\lambda_{n}}{2}\right|
$$

One can verify that in general the determinants (areas) in the integrands are invariant under the symmetry transformations of the $Z(s)$ function,

$$
x_{1} \rightarrow 1-x_{1} ; \alpha_{n} \rightarrow 1-\alpha_{n} ; y_{1} \rightarrow-y_{1} ; \beta_{n} \rightarrow-\beta_{n}
$$

and, of course, under complex conjugation $y_{1} \rightarrow-y_{1}$ and $\beta_{n} \rightarrow-\beta_{n}$ leaving the $x$-values fixed. Therefore, all the points in the vertices of the rectangles have identical statistical weights (triangle area-average) as they should.

To sum up, the area-average of all the triangles associated with a given zero $1 / 2+\lambda_{n}$, for $n=1,2, \ldots N$, which are enclosed by a single square $D_{N}$ of sides $2 L_{N}=2 \lambda_{N+1}$, centered at the center of symmetry $1 / 2+i 0$, is given by:

$$
\left\langle A\left(\lambda_{n} ; L_{N}\right)\right\rangle=\frac{\int_{D_{N}}\left|x_{1}\left(2 y_{1}-\lambda_{n}\right)-y_{1}+\frac{\lambda_{n}}{2}\right| d x_{1} d y_{1}}{2 \int_{D_{N}} d x_{1} d y_{2}} .
$$

To evaluate the integral of the absolute values of the determinants, the simplest way to proceed is by simply performing a change of a variables $x_{1} \rightarrow$ $x_{1}+1 / 2=x_{1}^{\prime}$, leaving $y_{1}$ fixed, of unit Jacobian, such that the integrand is now simplified to be just the absolute value of a product, which is equal to the product of absolute values, $\left|x_{1}^{\prime}\right| \times\left|2 y_{1}-\lambda_{n}\right|$. The $x^{\prime}$ integration is now between the limits $\left[1-L_{N}, 1+L_{N}\right]$ and the $y$-integration is between $\left[-L_{N} ; L_{N}\right]$. Upon splitting these domains of integration as a result of taking the absolute values yields for the triangle area-averages:

$$
\begin{gathered}
\frac{1}{8 L_{N}^{2}}\left(\int_{0}^{1+L_{N}} x_{1}^{\prime} d x_{1}^{\prime}-\int_{1-L_{N}}^{0} x_{1}^{\prime} d x_{1}^{\prime}\right)\left[\int_{\lambda_{n} / 2}^{L_{N}}\left(2 y_{1}-\lambda_{n}\right) d y_{1}-\int_{-L_{N}}^{\lambda_{n} / 2}\left(2 y_{1}-\lambda_{n}\right) d y_{1}\right]= \\
\left\langle A\left(\lambda_{n} ; L_{N}\right)\right\rangle=\frac{1+L_{N}^{2}}{4 L_{N}^{2}}\left(L_{N}^{2}+\frac{\lambda_{n}^{2}}{4}\right),
\end{gathered}
$$

where the cutoff $L_{N}=\lambda_{N+1}$. As expected these area-averages are symmetric functions in $\lambda_{n}$ because the statistical weights of pairs of complex conjugates are the same. Notice the correct units. The 1 in the factor $1+L_{N}^{2}$ has area-units 
such that it cancels the units of $L_{N}^{2}$ in the denominator leaving an expression with an overall dimension of area.

The integral domain where one evaluates all these area-averages, for $n=$ $1,2, \ldots N$ is chosen to be a single square $D_{N}$ that encloses the zeros $1 / 2+$ $i \lambda_{1}, 1 / 2+i \lambda_{2}, \ldots, 1 / 2+i \lambda_{N}$ as well as the ensemble of all triangles associated with each particular zero and whose area-average is given by the ratio of the two integrals. The square domain $D_{N}$ has sides $2 L_{N}$ where the cutoff scale is taken to be an explicit function of the cutoff value for $n=N$ as follows: $\lambda_{N}<L_{N} \leq \lambda_{N+1}$ and the square $D_{N}$ is centered at the center of symmetry $1 / 2+i 0$ of the $Z(s)=Z(1-s)$ function. Notice that the single square domain $D_{N}$ encloses (besides the triangles) exactly $N$ pairs of zeros, $N$ zeros with their complex conjugates; i.e. a total of $2 N$ zeros symmetrically located with respect the horizontal axis. For this reason, due to this mirror symmetry under complex conjugation, the square domain $D_{N}$ is the same as the square domain $D_{-N}$. The zeros $1 / 2 \pm i \lambda_{N+1}$ live on the boundary of the square domain $D_{N}=D_{-N}$ and are not counted.

After one has evaluated the triangle area-averages, which is how we assign the statistical weights to each of the zeros, the next goal is to compute $P\left(R_{N}\right)$ the probability of finding a zero inside the region $R_{N}$ in the complex plane. The region $R_{N}$ must not be confused with the original domain $D_{N}$ used to define the triangle area-averages in the first place. For example, we could take for the region $R_{N}$ a rectangle symmetrically distributed with respect to the critical line, of area $\left(2 L_{1}\right)\left(2 L_{2}\right)$ and take the double scaling limit: $L_{1} \rightarrow 0 ; L_{2} \rightarrow \infty$ such that the area of those domains remains fixed: $\left(2 L_{1}\right)\left(2 L_{2}\right)=A=$ constant. As the rectangles $R_{N}$ become more narrow and more elongated, they aproach asymptotically the critical line $1 / 2+i y$ (while the width collapses to zero).

One can also envision not taking the double-scaling limit for the regions $R_{N}$, but instead taking rectangular regions of increasing areas $\left(2 L_{1}\right)\left(2 L_{2}\right)$ where the width $2 L_{1}$ is increased until it reaches the unit width of the critical strip while its height $2 L_{2}$ approaches infinity. These rectangular regions are useful because they avoid intruding into the negative $X$-axis regions where the trivial zeros $s=-2,-4, \ldots-2 n$ are located.

Naturally as the sides of the square domains $D_{N}$ increase they will intrude into those regions of the complex plane where the trivial zeros of $\zeta(s)$ are located. At this stage it does not matter how we choose the domain $D_{N}$ to define a triangle area-average associated with a zero $1 / 2+i \lambda_{n}$, or for that matter, another point in the critical line $1 / 2+i y$. It does not matter if this square domain $D_{N}$ intrudes into those regions where the trivial zeros live. What matters comes afterwards when we define the regions $R_{N}$ in the complex plane where we wish to compute the probability that a zero is found. These regions $R_{N}$ must be chosen appropriately so that they will not enclose areas where the trivial zeros $s_{n}=-2 n+i 0$ are located. The best suited regions $R_{N}$ are indeed rectangular domains inside the critical strip, of fixed widths and increasinly larger heights. Or with narrower widths, such that in the asymptotic limit, the region $R_{\infty}$ collapses into the critical line $1 / 2+i y$.

To simplify our calculations, and to answer the question if the $\mathrm{RH}$ is true, 
we shall take a simpler route and choose directly a region $R_{N}$ given already by a line interval in the critical line (instead of a rectangular domain of nonzero width). In the large $N$ limit the region $R_{N}$ will coincide by definition with the full critical line $1 / 2+i y$.

The first step has already been taken when we evaluated the triangle areaaverages from the triangle-picking formula based on geometric probability, using a single square domain $D_{N}$, at each time, with sides $2 L_{N}$ and with cutoffs $\lambda_{N}<L_{N}=\lambda_{N+1}$. The second step to be taken after obtaining the area-average associated with a given zero $\left\langle A\left(\lambda_{n} ; L_{N}\right)\right\rangle$, and for that matter, associated with all the points in the critical line $\left\langle A\left(y, L_{N}\right)\right\rangle$ whose $y$ coordinates, at each time, are bounded by $-\lambda_{N+1} \leq y \leq \lambda_{N+1}$ is when we go ahead and ask what is the geometric probablity $P\left(R_{N}\right)$ to find a zero inside a region $R_{N}$ given by a line interval in the critical line (instead of a rectangular domain of nonzero width) whose $y$-values are also bounded by the similar conditions: $-\lambda_{N+1} \leq y \leq \lambda_{N+1}$.

These regions $R_{N}$, whether they are line intervals in the critical line, or rectangular domains of narrower widths and larger heights inside the critical strip, do not contain the trivial zeros $s_{n}=-2 n+i 0$. Since, the HadamardValle de la Poussin theorem states that there are no zeros in the vertical lines whose real parts are 0,1 , this means that we do not have to worry about these vertical line boundaries of the critical strip.

Therefore, the probability to find a zero in (all of) the critical line, i.e the probability that the $\mathrm{RH}$ is true, is given by the $N=\infty$ limit of $P\left(R_{N}\right)$ !. The crucial question is: Despite that we have a well defined algorithm, as we will show below, to define $P\left(R_{N}\right)$, can we compute such large $N$ limit of $P\left(R_{N}\right)$ with absolute certainty? If the $N=\infty$ limit of $P\left(R_{N}\right)$ is unity this means that the probablity to find a zero in the critical line is 1 , and hence the $\mathrm{RH}$ is true. If the probablity is not unity in the large $N$ limit, then the $\mathrm{RH}$ is false because this means that there is a finite nonzero probablity of finding a zero outside the critical line. We will return to this crucial issue below.

\subsection{The probability for the $\sinh (\mathrm{s})$ case}

To verify the validity of this construction to the $\mathrm{RH}$, we will apply firstly such geometric probability techniques for the zeros of $\sinh (s)$ all of which we know lie at equally-spaced intervals of $\pi$ in the vertical (imaginary) $Y$-axis, $s_{n}=0+i n \pi$. Thus the location of the zeros of the $\sinh (s)$ behave exactly as the location of the zeros of zeta. Both zeros live in (different) vertical lines irrespective of their spacings. In Section 3 we showed why the $\sinh (s)$ function is also related to the inner of products of eigenfunctions of (other) differential operators (which are not self adjoint) obeying similar symmetry properties with respect the vertical (imaginary) axis as the function $Z(s)$ does with respect the critical Riemann line. For this reason we can aply this probabilistic method to both the $Z(s)$ and $\sinh (s)$ functions to evaluate the triangle area-average:

$$
\left\langle A\left(\alpha_{n}+i \beta_{n}\right)\right\rangle=\langle A(0+i n \pi)\rangle=\frac{\int A(\text { triangle }) d x_{1} d y_{1}}{\int d x_{1} d y_{1}},
$$


where the 3 points coordinates, $\left(x_{1}, y_{1}\right) ;\left(x_{2}, y_{2}\right) ;(x, y)$, in the $\sinh (s)$ case obey the different relations $s=s_{1}^{*}+s_{2}=0+i \beta_{n}$ (see Section 3):

$$
\begin{gathered}
x_{1}+x_{2}=x=0 \Rightarrow x_{2}=-x_{1}, \\
y_{2}-y_{1}=\beta_{n}=n \pi \Rightarrow y_{2}=y_{1}+\beta_{n}=y_{1}+n \pi,
\end{gathered}
$$

the areas inside the integral are the absolute values of $1 / 2$ times the determinant:

$$
\frac{1}{2}\left|x_{1}\left(2 y_{1}-\beta_{n}\right)\right|=\frac{1}{2}\left|x_{1}\right|\left|2 y_{1}-\beta_{n}\right|,
$$

this factorization of the absolute values facilitates the evaluation of the integrals as the product of two integrals:

$$
\int_{-L_{N}}^{L_{N}}\left|x_{1}\right| d x_{1}=2 \int_{0}^{L_{n}} x_{1} d x_{1}=L_{N}^{2}
$$

and the $y_{1}$ integral of $\left|2 y_{1}-\beta_{n}\right|$ must be split into two separate integrals:

$$
\int_{\beta_{n} / 2}^{L_{N}}\left(2 y_{1}-\beta_{n}\right) d y_{1}-\int_{-L_{N}}^{\beta_{n} / 2}\left(2 y_{1}-\beta_{n}\right) d y_{1}=2\left(\frac{\beta_{n}^{2}}{4}+L_{N}^{2}\right) .
$$

Collecting all the terms stemming from the $x_{1}, y_{1}$ integrals, including the extra factor of $1 / 2$ in front of the determinant, and dividing by the area $\left(2 L_{N}\right)^{2}$ of the square domain $D_{N}$ of side $2 L_{N}$, one finally obtains the average area of all the triangles associated with the given zero $0+i \beta_{n}$ and which fall inside the square domain of area $\left(2 L_{N}\right)^{2}$ :

$$
\left\langle A\left(\beta_{n} ; L_{N}\right)\right\rangle=\frac{1}{4}\left(\frac{\beta_{n}^{2}}{4}+L_{N}^{2}\right),
$$

we obtained, as expected, a symmetric function, $\left\langle A\left(-\beta_{n} ; L_{N}\right)\right\rangle=\left\langle A\left(\beta_{n} ; L_{N}\right)\right\rangle$, which states that the statistical weights of any pairs of complex conjugates is the same. The imaginary parts of the zeros of $\sinh (s)$ are, $\beta_{n}=n \pi$. These square domain $D_{N}=D_{-N}$ of sides $2 L_{N}$ have their centers located at the center of symmetry $0+i 0$, for the $\sinh (s)$ case, and contain $N$ pairs zeros. The sides $2 L_{N}$ are given by the cutoff scale: $2 L_{N}=2(N+1) \pi>2 N \pi$ to ensure that the single square domain encloses exactly $N$ pairs of zeros, $0+i n \pi$ (and complex conjugate) for those values of $n$ ranging from $1,2, \ldots N$.

Now that we have evaluated the average area of the triangles associated with a given zero $0+i \beta_{n}$ inside a square domain of sides $2 L_{N}$ and centered at $0+i 0$ as an even function of the number $\beta_{n}=n \pi$ and the cutoff scale $L_{N}=(N+1) \pi$, we may compute the probability $P\left(R_{N}\right)$ to find a zero in the region $R_{N}$ in the vertical $Y$-axis.

This is achieved by defining the $P\left(R_{N}\right)$ once again in terms of geometric probability, as the ratio of areas. Given the sequence of $N$ triangle area-averages, 
associated with the sequence of $0+i \beta_{1}, 0+i \beta_{2}, \ldots 0+i \beta_{N}$ zeros, plus complex conjugates, we shall take their discrete sum over the triangle area-averages evaluated at the $N$ discrete number of pairs of zeros $0+i \beta_{n}$ (plus their complex conjugates) and then divide by the integral of the continuous distribution of area-averages $\int\left\langle A\left(y, L_{N}\right)\right\rangle d y$ associated with all the points in the $Y$-axis from $-(N+1) \pi \leq y \leq(N+1) \pi$. This integral can be written as:

$$
I\left(R_{N}\right)=\int_{-(N+1) \pi}^{(N+1) \pi}\left\langle A\left(y ; L_{N}\right)\right\rangle d y=\frac{1}{4} \int_{-(N+1) \pi}^{(N+1) \pi}\left[\frac{y^{2}}{4}+(N+1)^{2} \pi^{2}\right] d y
$$

We will explain in detail next why the geomeric probability to find a zero inside the region $R_{N}$, a line interval of length $2 L_{N}$ in the imaginary axis, is related to the study of the following fractions:

$$
P\left(R_{N}\right)=\frac{\sum_{n=-N}^{N}\left\langle\Delta_{n}\right\rangle\left\langle A\left(\beta_{n} ; L_{N}\right)\right\rangle}{\int_{-(N+1) \pi}^{(N+1) \pi}\left\langle A\left(y ; L_{N}\right)\right\rangle d y} .
$$

Because all the zeros $0+i \beta_{n}$ are equally-spaced, the average separation $\left\langle\Delta_{n}\right\rangle$ betwen consecutive zeros is $\pi$ :

$$
\left\langle\Delta_{n}\right\rangle=\frac{1}{N} \sum_{n=1}^{N}\left(\beta_{n+1}-\beta_{n}\right)=\frac{(N+1) \pi-\pi}{N}=\pi .
$$

Notice that one can always scale all the zeros $\beta_{n}$ by dividing by $\pi$, and in doing so, the mean spacing is equal to 1 . However, this will not be the case for the average separation of consecutive Riemann zeros. The mean-spacing among the zeta zeros; i.e the average number of zeros in a given interval is basically the number of zeros divided by the size of the interval in the limit that $N=\infty$. Taking the interval to lie between $\left[0, \lambda_{N}\right]$ the mean-density of zeros in that interval is approximately (to leading order) given by the celebrated formula [3]:

$$
\left\langle\rho_{N}\right\rangle=\frac{N}{\lambda_{N}} \sim \frac{\frac{\lambda_{N}}{2 \pi} \log \frac{\lambda_{N}}{2 \pi}}{\lambda_{N}}=\frac{1}{2 \pi} \log \frac{\lambda_{N}}{2 \pi} .
$$

This is the place where random matrix theory overlaps with our geometric probability (stochastic geometry) approach to the RH and perhaps the underlying reason why random matrix models are deeply related to the RH. As far as we know this reason is not known, it was an empirical fact that the Montgomery pairs correlation distribution of zeros agreed with Dyson's results based on random matrix models.

By symmetry (complex pairs have equal statistical weights) we can just take the sums and integrals from $[1, N]$ and $[0,(N+1) \pi]$, respectively, since the 
common factors of 2 cancel out in the ratio. Notice that the zeta function $\zeta(s)$ and the function $Z(s)$ does not have a zero at the center of symmetry $s=1 / 2+i 0$. In Section 3 we proved that the inner products (of different eigenfunctions) is given by the function $G(s)=\sinh (s) / s$ that does not have a zero at $s=0+i 0$ (center of symmetry in this case) because $G(s=0+i 0)=1 \neq 0$. Therefore, we can safely take the sums from 1 to $N$ and the integral from 0 to $N+1$ in units of $\pi$ without counting the point $0+i 0$ (it lives in the boundary of the region $R_{N}$ ).

The reader may notice that because the zeros of the function $\sinh (s)$ are all equally spaced $\beta_{n}=n \pi$ in units of $\pi$, and that the areas grow as $n^{2} \pi^{2}$, the large $N$ limit of the discrete sum is precisely given by the integral in the denominator and the answer for $P\left(R_{N}\right)$ in the large $N$ limit is going to be 1 . To show this, we will sum the discrete series and perform the integral. The discrete sum

$$
S\left(R_{N}\right)=\frac{1}{4} \pi^{3} \sum_{n=1}^{N}\left[\frac{n^{2}}{4}+(N+1)^{2}\right]
$$

gives a straightforward result by simply summing the quadratic series:

$$
\sum_{n=1}^{N} n^{2}=\frac{N(2 N+1)(N+1)}{6}
$$

so that

$$
\begin{gathered}
S\left(R_{N}\right)=\frac{1}{4} \pi^{3}\left[\frac{N(2 N+1)(N+1)}{24}+N(N+1)^{2}\right]= \\
\frac{1}{4} \pi^{3} \frac{24 N(N+1)^{2}+N(N+1)(2 N+1)}{24}=\frac{1}{4} \pi^{3}(N+1) \frac{26 N^{2}+25 N}{24} .
\end{gathered}
$$

The integral (in units of $\pi$, the basic cell size) in the denominator yields:

$$
\begin{gathered}
I\left(R_{N}\right)=\frac{1}{4} \int_{0}^{(N+1) \pi}\left[\frac{y^{2}}{4}+(N+1)^{2} \pi^{2}\right] d y= \\
\frac{1}{4} \pi^{3}\left[\frac{(N+1)^{3}}{12}+(N+1)^{3}\right]=\frac{1}{4} \pi^{3}(N+1) \frac{26(N+1)^{2}}{24} .
\end{gathered}
$$

The ratio of the sum $S\left(R_{N}\right)$ and the integral $I\left(R_{N}\right)$ is then:

$$
P\left(R_{N}\right)=\frac{26 N^{2}+25 N}{26(N+1)^{2}}<\frac{26 N^{2}+26 N}{26(N+1)^{2}}=\frac{N}{N+1}<1 .
$$

The fact that the probability $P\left(R_{N}\right)$ to find a zero inside the interval $R_{N}$ is less than unity is consistent because $P\left(R_{N}\right)$ cannot exceed unity. In the $N \rightarrow \infty$ the quantity

$$
P\left(R_{N} \rightarrow \infty\right)=\frac{26 N^{2}+25 N}{26(N+1)^{2}} \rightarrow\left(\frac{N}{N+1}\right)^{2} \rightarrow 1
$$


since the leading powers $N^{2}$ dominate, and as expected the $P\left(R_{N}\right)$ to find a zero in the large $N$ limit, when the measure of the region $R_{N}$ goes to infinity, is exactly equal to 1 for the $\sinh (s)$ case. Concluding: the probability to find a zero in the imaginary axis is unity as expected. The probability to find a zero outside the imaginary axis is zero.

A very important remark is now in order. One could as well have computed the numbers $P\left(R_{N}\right)$ by taking for the increment $\Delta=\pi$, the values of $\Delta=1$ instead, to compute the ratios of the sums $\sum\left\langle A_{n}\right\rangle$ divided by the integrals $\int\langle A(y)\rangle d y$. In this case what one would have obtained in the large $N$ limit is the mean-density of zeros of $\sinh s$; i.e the number of zeros per unit length which is equal to $\langle\rho\rangle=1 / \pi$.

This result makes perfect sense because one has one zero in the interval $[0, \pi]$; two zeros in the interval $[0,2 \pi] ; \ldots N$ zeros in the interval $[0, N \pi]$. Hence, the mean-density of zeros is $N /(N \pi)=1 / \pi$. By multpliying the mean-density by the mean-spacing between zeros $\langle\Delta\rangle=\pi$, one recovers back $P=(1 / \pi) \pi=1$. We shall return to this issue of calculating the mean-density of zeros for the $\zeta(s)$ below in connection to the evaluation of the probability to find a nontrivial zeta zero in the critical line.

It is essential to emphasize that it would be meaningless to try to derive the probability by comparing the measure space of the discrete number of zeros (zero Lebesgue measure) with the nonzero measure of the real line. We are not asking what is the probability (zero in that case) that upon throwing a dart it will strike a zero! What we have done is to count the number of ways in which one can generate a zero from the inner products of two states associated with a pair of complex numbers, $s_{1}, s_{2}$ and then divided by the number of all possible pairings inside a given region and taking the size of those regions to infinity when we take the large $N$ limit. We performed the counting by means of geometric probability; i.e by computing the area-averages of an infinite family of triangles associated to each point $s$ in the critical lines, whether it is a zero or not.

The reader may ask the question: Do the numbers $P\left(R_{N}\right)$ depend on the shape of the domains $D_{N}$ used to compute the triangle-area-averages? There is no reason why numbers $\left\langle A\left(\lambda_{n} ; L_{N}\right)\right\rangle$ (square domain) should be the same as the numbers $\left\langle A\left(\lambda_{n} ; L_{1}, L_{2}\right)\right\rangle$ obtained for a rectangular domain of area $\left(2 L_{1}\right)\left(2 L_{2}\right), \ldots$ What one should expect is that the large $N$ limit of the two different series $P\left(R_{N}\right)$, based on different domains to compute the triangle area-averages, should be the same. Both series should converge to the same large $N$ limit. If the large $N$ limit depends on the shape of the domains used to compute the triangle area-averages this will be a great surprise, which implies that the large $N$ limit is not unique. We are assuming that the large $N$ limit is unique and truly independent of the shape of the domains, however, this has to be proved. The large $N$ limit of the algebra $S U(N)$ is basis-dependent. In one particular basis, Jens Hoppe [34] long ago has shown that $S U(\infty)$ algebra is isomorphic to the infinite-dim algebra of area-preserving diffs of a sphere.

Therefore, concluding the calculation for the zeros of the $\sinh (s)$ function, the probability to find a zero (and a discrete infinity of zeros) of the $\sinh (s)$ 
function in the region $R_{\infty}$ given by the imaginary axis $-\infty<y<+\infty$ is indeed unity as expected. This result occurs because all the zeros are equally spaced in units of $\pi$, therefore the continuous integral matches the discrete sum in the $N=\infty$ limit.

\subsection{The Riemann zeta zeros case}

Let us return to the computation of the probabilities $P\left(R_{N}\right)$ to find a Riemann zeta zero in the region $R_{N}$ corresponding to a finite interval in the critical line $1 / 2+i y$ given by $-\lambda_{N+1} \leq y \leq \lambda_{N+1}$. Due to the symmetry under complex conjugation that requires that the statistical weights for any pair of complex conjugates be the same, we can divide the line interval in half and focus soley in the upper portion of the critical line. Because the imaginary parts of the zeta zeros $\lambda_{n}$ are not equally spaced as the zeros of the $\sinh (s)$ function, one cannot conclude with absolute certainty that the integral in the denominator matches precisely the discrete sum in the $N=\infty$ limit.

In the Riemann case, due to the fact that the mean-spacing of the zeros is highly non-trival, it is more convenient to study the large $N$ limit of the ratios of the quantities studied above by setting the $\Delta=1$ which will furnish the density of zeros $\mathcal{D}\left(R_{N}\right)$ expressed in terms of geometric probability theory as follows:

$$
\mathcal{D}\left(R_{N}\right) \equiv \frac{\sum_{n=1}^{N}\left(\lambda_{N+1}^{2}+\frac{\lambda_{n}^{2}}{4}\right)}{\int_{0}^{\lambda_{N+1}}\left(\lambda_{N+1}^{2}+\frac{y^{2}}{4}\right) d y} .
$$

The quantity $\mathcal{D}\left(R_{N}\right)$ is a density of zeros and has the correct units (dimensions) of an inverse length. To show why one can first look at the asymptotic behaviour of this density by rewriting the above expression as:

$$
\mathcal{D}\left(R_{N}\right) \equiv \frac{N}{\lambda_{N+1}} \frac{\frac{1}{N} \sum_{n=1}^{N} A_{n}}{\frac{1}{\lambda_{N+1}} \int_{0}^{\lambda_{N+1}} A(y) d y} .
$$

Where the numerator is now the discrete average $\left\langle A_{n}\right\rangle$ of the $N$-numbers $A_{n}$, and the denominator is now just the mean-value of the function $A(y)$ in the interval $\left[0, \lambda_{N+1}\right]$. In the asymptotic regime (large $N$ limit), the discrete averages should not differ much from the mean-values and one obtains that, asymptotically, the density of zeros obtained from geometric probability $\mathcal{D}\left(R_{N}\right)$ behaves as one would expect:

$$
\mathcal{D}\left(R_{N}\right) \sim \frac{N}{\lambda_{N+1}} .
$$


Notice that this behaviour only holds in the asymptotic regime and not necessarily in the intermediate regime of finite values of $N$. Thus, there is no reason why, a priori, without doing the full calculations, that the density of zeros obtained from geometric probability $\mathcal{D}\left(R_{N}\right)$, in the intermediate regime of finite values of $N$, will have to coincide with $\left(N / \lambda_{N+1}\right)$ nor with the density of zeros given by the celebrated formula assuming the $\mathrm{RH}$ is true and that all the zeros are simple.

Notice that upon using the asymptotic approximation given by (63, after straightforward algebra, one can deduce from $(61,63)$ a recursion formula for the $N+1$-th zero $\lambda_{N+1}$ in terms of the $N$ previous zeros. The quantity $\lambda_{N+1}^{2}$ is approximately equal to three times the average of the squares of the previous $N$ zeros!:

$$
\lambda_{N+1}^{2} \sim 3\left\langle\lambda_{n}^{2}\right\rangle=\frac{3}{N} \sum_{n=1}^{N} \lambda_{n}^{2} .
$$

Another recursive relation is obtained from $\mathcal{D}_{N} \sim[1 /(2 \pi)] \log \left[\lambda_{N+1} /(2 \pi)\right]$. As far as we know these recursion relations, derived from the geometric probability density $\mathcal{D}_{N}$, have not been obtained before. This corroborates further the validity of our method.

If the RH is true and all the zeros are simple, the mean number-density of zeta zeros in the interval $\left[0, \lambda_{N+1}\right]$; i.e. the number of zeros per unit length (in that interval) is given (to leading order) by the celebrated formula:

$$
\left\langle\rho_{N+1}\right\rangle=\frac{N+1}{\lambda_{N+1}} \sim \frac{1}{2 \pi} \log \frac{\lambda_{N+1}}{2 \pi} .
$$

A more general and fundamental result, known for a long time, is the asymptotic behaviour of the density of zeros inside the critical strip. If $N(T)$ denotes the mumber of zeros $s_{o}=\beta+i \gamma$ in the critical strip with ordinates $0<\gamma \leq T$, then as $T$ goes to $\infty$, the number of zeros is [3]:

$$
N(T)=\frac{T}{2 \pi}\left(\log \frac{T}{2 \pi}-1\right)+O(\log T) .
$$

If the $\mathrm{RH}$ is true, and the zeros are simple, then one can equate in this special case the number $N(T)$ with the number of zeros $N_{1 / 2}(T)$ in the interval of the critical line whose height is given by $T$.

However, if the $\mathrm{RH}$ is not true, it is clear why then $N_{1 / 2}(T)<N(T)$, and consequently we could define properly the probablity to find a zero in the critical line by the ratio of the quantities in the $T=\infty$ limit:

$$
P=\lim _{T \rightarrow \infty} \frac{N_{1 / 2}(T)}{N(T)} .
$$

Hardy, Littlewood, Selberg, Levinson, Conrey found general estimates of the ratios of $N_{1 / 2}(T) / N(T)=C<1$. Two estimates of the contants $C<1$ obtained by Levinson and Conrey were $1 / 3 ; 2 / 5$ respectively. For historical details about these numerical bounds on the ratio of the number of zeta zeros in the critical 
line with respect to the number of zeros in the critical strip, mean-value theorems and random matrix theory see [57].

To sum up, one may write $N_{1 / 2}(T)=C N(T)$ where $C \leq 1$ and assign the number $C \leq 1$, in the limit $T \rightarrow \infty$, as the correct probablity to find a zero in the critical line. In order to find what the value of $C=P$ is, we shall be comparing the density of zeros in the critical line obtained from geometric probability with the density of zeros, assuming the $\mathrm{RH}$ is true and that the zeros are simple. If there is a very good match between both densities of zeros in the large $N$ limit, this is a signal that the $\mathrm{RH}$ is most likely to be true. If there is a perfect match in the large $N$ limit, the $\mathrm{RH}$ is true. If there is a clear mismatch, let us say in the ratio of the two densities; i.e. the ratio differs considerably from 1 , then the $\mathrm{RH}$ is not true.

The negative case scenario is better visualized if one sets from the vey beginning that the number of zeros in the critical line is a fraction of the number in the critical strip: $N_{1 / 2}(T)=C N(T)$, where $C=P \leq 1$. and then one plots the one-parameter family of graphs of the numbers $P_{C}\left(R_{N}\right)$ parametrized by the constants $C$ :

$$
P_{C}\left(R_{N}\right)=\frac{2 \pi}{C \log \frac{\lambda_{N+1}}{2 \pi}} \mathcal{D}_{N},
$$

where $\mathcal{D}_{N}$ are the densities of zeros obtained from the geometric probablity expresions (61). There are now three curves as functions of $N$ which need to be to studied in the large $N$ limit:

$$
\mathcal{D}_{N} ; \frac{N}{\lambda_{N+1}} ; \frac{C}{2 \pi} \log \frac{\lambda_{N+1}}{2 \pi},
$$

which asymptotically should approach each-other, for a given value of $C$.

The key question to ask is what is the value of $C \leq 1$ which gives the most precise asymptotic match between $\mathcal{D}_{N}$ and $[C /(2 \pi)] \log \left[\lambda_{N+1} /(2 \pi)\right]$ ?. If one includes higher order corrections to the number-density of zeros, to plot the graphs, then one can also ask which value of $C$ gives the most precise match in the intermediate regions where $N=$ finite?. If the answer for both asymptotic and intermediate regions is $C=P=1$, the $\mathrm{RH}$ is true. If $C=P<1$, the $\mathrm{RH}$ is not true. The case $C=P>1$ must be disregarded since the probablity to find a zero in the critical line cannot be greater than 1 .

In the $\sinh (s)$ we found that the large $N$ limit of the density of zeros $\mathcal{D}\left(R_{N}\right)$ (obtained from geometric probability) was $1 / \pi$. Since the average spacing $\left\langle\Delta_{N}\right\rangle=\pi$, for all values of $N$, then $P=(1 / \pi)\left\langle\Delta_{N}\right\rangle=(1 / \pi) \pi=1$ as expected.

Notice that for finite values of $N$, it is more reasonable to write the higher order corrections of the number of zeta zeros given by the main formula,

$$
N=\frac{\lambda_{N}}{2 \pi}\left(\log \frac{\lambda_{N}}{2 \pi}-1\right)+\log \frac{\lambda_{N}}{2 \pi}+\ldots
$$

but for simplicity we concentrate on the leading terms for now.

In essence what this geometric probability approach suggests is that it sufficient to study the behaviour of the zeros in the critical lines because they 
seem to encode global information about the algebraic behaviour of the functions $\sinh (s)$ and $\zeta(s)$ in their critical strips. Not unlike index theory (K theory) whereby the number of zero modes of a Dirac operator encodes topological information of a manifold.

The reader may ask how do we handle the fact that we have a truly random sequence (series) of numbers $P_{C}\left(R_{N}\right)$ ?. To know all the infinite random numbers $\lambda_{n}$ all the way to infinity is not a feasable task. Despite this intrinsic randomness how can we decide with full certainty whether the $\mathrm{RH}$ is true with probability one? Do we have another example of what has been called a random truth that cannot be proved, in the spirit of Godel's undecidable propositions (incompleteness theorem). Chaitin has made some arguments in favour of this idea of randomness in connection to the $\mathrm{RH}$ [35]. It is very important to emphasize that we have a well defined algorithm to generate the random numbers $P_{C}\left(R_{N}\right)$, for each values if $C \leq 1$, because we know precisely the functional form of the triangle area-averages. What we don't know is a general formula to generate the zeros $1 / 2+i \lambda_{n}$, for any $n$. The way to handle this dilemma to get a flavor of the behaviour of the fractions $P_{C}\left(R_{N}\right)$ for $N=1,2, \ldots \infty$ is to go ahead and use the tables of the known zeros. Tables up to the zero number $10^{22}$ have been provided by Odlyzko. In particular, to find out the scaling behaviour of the sequence of random numbers $P_{C}\left(R_{N}\right)$. Therefore, it is important to find out the scaling behaviour of the fractions.

The reason we should study the scaling behaviour of the fractions $P_{C}\left(R_{N}\right)$ despite the random nature of the zeros is because there may exist a quasiperiodic long-range order. Wolf [36] has studied the multi-fractal patterns in the prime-numbers distribution so one would expect some sort of self-simililarity (although not strictly so) in the scaling behaviour of the random fractions $P_{C}\left(R_{N}\right)$ because the distribution of zeta zeros is tightly connected to the distribution of primes resulting from the Hadamard-Euler product expansion of the zeta function.

One of us suggested [37] a while ago that there must be a deep reason why the spectral properties associated with the binary Fibonnaci sequence of numbers (the hallmark of quasi-periodic long range order, golden mean route to chaos, etc...) is related to the function $\sin ^{2}(\pi x) /(\pi x)^{2}$ which also turns out in the Montgomery-Dyson pair-correlations of the imaginary parts of the zeta zeros in random matrix theory.

For this reason, in Section 5 we will study a fractal SUSY QM model (fractal Schroedinger equation) with a Weierstrass potential, such that the shape of the potential is a fractal curve of dimension $D=1.5$ and which generates all the $\lambda_{n}$ for its spectrum. This is nothing but the implementation of the HilbertPolya idea to solve the RH. The fractal dimension $D=1.5$ of the Weierstrass potential is precisely the value that is connected to the $1 / f$ noise spectrum, as we shall see. Long ago Franel-Landau proved that the $\mathrm{RH}$ is related to an arithmetic statement connected to the Farey sequence of fractions [23], and not suprisingly, it is the scaling behaviour of the fractions $P_{C}\left(R_{N}\right)$ that one would wish to analyse.

The fact that we know that the $P\left(R_{N}=\infty\right)$ is unity with absolute certainty 
for the zeros of the $\sinh (s)$ function, and whose location along the imaginay $Y$-axis mimics perfectly the behaviour of the Riemann zeros in the critical line, corroborates that evaluating the geometric probability whether the $\mathrm{RH}$ is true or not, is the right procedure to take and the right question to ask. For rigorous work concerning the plausible reasons to doubt the $\mathrm{RH}$ see Ivic [38]. In this negative case scenario, one should have computed the probability that the zeros fall inside a certain rectangular domain within the critical strip instead of line intervals.

The crux of this section is based in writing the Riemann fundamental function $Z(s)$ in terms of "inner" products, $\left\langle\psi_{s_{1}} \mid \psi_{s_{2}}\right\rangle=\left\langle\psi_{s_{o}} \mid \psi_{s}\right\rangle=Z(s)$, or pairings among two complex numbers $s_{1}, s_{2}$ to give a third number $s=s_{1}^{*}+s_{2}-1 / 2$, such that $S\left(s_{1}, s_{2}\right)=Z(s)$ to be more precise. Then we proceeded to "count" in how many ways one can generate a zero $s$ from the two complex numbers $s_{1}, s_{2}$. This is achieved via geometric probability theory that allows us to find the explicit functional form of the triangle-area-average function $\left\langle A\left(y ; L_{n}\right)\right\rangle$ for any point in the critical line, and of course, $A\left(\lambda_{n} ; L_{N}\right)$, associated with the area-averages of the infinite number of triangles whose 3 vertices are located at the triplet of points $\left(s_{1}, s_{2}, s\right)$.

Next, these triangle-area-averages are employed to evaluate the density of (simple) zeros $\mathcal{D}_{N}$ in the critical line and which must be compared with the alledged density of zeros in the critical strip based on the celebrated formula $[1 /(2 \pi)] \log \left[\lambda_{N+1} /(2 \pi)\right]$, in the large $N$ limit, assuming that the RH is true and that all the zeros are simple. If these two densities truly match then this is a signal that the $\mathrm{RH}$ is true. If they do not match this is a signal that the $\mathrm{RH}$ is not true.

In Section 4.4 we will analyse in detail the numerical results of the graphs of the numbers $P_{C}\left(R_{N}\right)$ as functions of $N$, and the implications of our findings. We will see that the $\mathrm{RH}$ is most likely to be true since the density of zeros obtained from geometric probability matches very well indeed, in the large $N$ limit, the density of zeros based on the celebrated formula, assuming the RH is true and that the zeros are simple.

Concluding, in general we are going to have three scenarios in the large $N$ limit:

1. Optimistic scenario; $P=1$, the $\mathrm{RH}$ is true, so the probability to find a zero and $m$ zeros in the critical line is 1 , even when $m=\infty$.

2. Surprising scenario: $P<1$, the $\mathrm{RH}$ is false since there is a finite nonzero probability of finding a zero (in mutiples of four) in the vertices of one rectangle, outside the critical line.

3. Shocking scenario: Undecidable answer due to an intrinsic element of randomness, we are unable to compute the $N \rightarrow \infty$ limit of the numbers $P_{C}\left(R_{N}\right)$, due to our lack of knowledge of all the Riemann zeros, that would have provided us with a satisfactory answer, with full and absolute certainty, whether the RH is true or not. 


\subsection{Numerical estimates of the random series $P\left(R_{N}\right)$}

Here we shall evaluate the ratios $P_{C}\left(R_{N}\right)$ based on the tables of zeros provided by Odlyzko. For simplicity we shall look at one graph for $C=1$. For this purpose we evaluate the numbers $P_{C}\left(R_{N}\right)$ as functions of $N$ and display the graphs in Figures 5 and 6 for the first $N=100000$ zeros of the tables of Odlyzko.

Asymptotically the expression for the density of zeros in the critical line $\mathcal{D}\left(R_{N}\right)$ derived from geometric probability (61) tends to :

$$
\frac{N_{1 / 2}\left(\lambda_{N+1}\right)}{\lambda_{N+1}}
$$

where $N_{1 / 2}\left(\lambda_{N+1}\right)$ is the number of zeros in the interval of the critical line $1 / 2+i y$ and whose height is $\lambda_{N+1}$.

The density of zeros obtained from the celebrated formula is asymptotically given by:

$$
\frac{N\left(\lambda_{N+1}\right)}{\lambda_{N+1}}=\frac{1}{2 \pi} \log \frac{\lambda_{N+1}}{2 \pi},
$$

where $N\left(\lambda_{N+1}\right)$ is the number of zeros in the rectangular region of the critical strip whose height is $\lambda_{N+1}$ and whose width is 1 . See Figure 4.

Thus, upon dividing these two last expressions and taking the large $N$ limit, one obtains the probability to find a zero in the critical line:

$$
\begin{gathered}
\lim _{N \rightarrow \infty} \frac{N_{1 / 2}\left(\lambda_{N+1}\right)}{N\left(\lambda_{N+1}\right)}= \\
\lim _{N \rightarrow \infty} P_{1}\left(R_{N}\right)=\lim _{N \rightarrow \infty} \frac{2 \pi}{\log \frac{\lambda_{N+1}}{2 \pi}} \mathcal{D}\left(R_{N}\right) .
\end{gathered}
$$

This last expression is the main formula we shall use, and upon writing the density of zeros $\mathcal{D}\left(R_{N}\right)$ from the main expression derived from geometric probability, we will plot the graphs $P_{1}\left(R_{N}\right)$ as functions of $N$ in Figure 5 in the regions from $N=1$ to $N=1000$, and from $N=1$ to $N=100000$, respectively.

At $N=10^{5}$ the curve $P_{1}\left(R_{N}\right)$ reaches the value of 1 . For higher values of $N$ we expect that the curve $P_{1}\left(R_{N}\right)$ will reach unity asymptotically and consequently, the $\mathrm{RH}$ is most likely to be true since the $P \rightarrow 1$ !. A more extensive computation will capture better the behaviour of $P_{1}\left(R_{N}\right)$ as one reaches the last zeros of the tables of Odlyzko, $N=10^{22}$. This will tell us more precisely whether the asymptote is indeed located at 1 (the $\mathrm{RH}$ is true with probability one) or below 1 !.

\subsection{A relation to random matrix theory and quantum gravity}

Instead of computing the geometric probability based on area-averages of a continuum of triangles associated with a given zero, one could start by writing 
down the table of pairs of random complex numbers as follows. Given a list of $N$ random complex numbers:

$$
s_{1}^{(1)}, s_{1}^{(2)}, \ldots s_{1}^{(N)},
$$

associated with the first element $s_{1}$, and the list of $N$ random complex numbers:

$$
s_{2}^{(1)}, s_{2}^{(2)}, \ldots s_{2}^{(N)},
$$

associated with the second element $s_{2}$, one then constructs the table of $N^{2}$ random complex entries given by the $N \times N$ complex matrix (not necessarily Hermitean) obtained from the composition rule

$$
s=s_{1} \otimes s_{2} \equiv s_{1}^{*}+s_{2}-1 / 2 .
$$

One can repeat this procedure for another list of $N$ pairs of numbers $\left(s_{1}, s_{2}\right)$ and in this fashion one constructs an infinite ensemble of $N \times N$ random complex matrices. Once can then find the (complex) eigenvalues $\gamma_{1}, \gamma_{2}, \ldots \gamma_{N}$ for each matrix within the ensemble, and ask what is the probability that there is a zero $1 / 2+i \lambda_{n}$ among that family of complex eigenvalues.

The large $N$ limit of this procedure should give us a measure of the probablity to find a Riemann zeta zero from the inner products of the pairs of numbers $s_{1}, s_{2}$. Since this method is based on a countably-finite combinations of pairs, it must not be confused with the evaluation of the geometric probability that was based on the computation of triangle-area averages involving integrals (continuum). Therefore, we could postulate why random matrix theory, in the large $N$ limit, could be just a discretization approximation to the geometric probability approach in solving the $\mathrm{RH}$. This discretization approach to the computation of triangle-area-averages based on random matrix theory is related to the large $N$ limit in discrete random triangulations in quantum gravity [40] and to the large $N$ matrix models in two-dimensional string theory when the Feynman path integral is approximated by discrete (regularized) sums. The relevance of the zeta function in phase spaces. Moyal star products and the evaluation of path integrals in QFT has been discused in [41].

\section{A fractal supersymmetric quantum mechani- cal model}

The Hilbert-Polya proposal to prove the $\mathrm{RH}$ is based on the possibility that the imaginary parts of the nontrivial zeros of zeta are the real eigenvalues of some unknown Hermitian operator [5]. If the nontrivial zeros of the Riemann zeta function are given by $s_{n}=1 / 2+i \lambda_{n}$, and if there exists a suitable Hermitian operator $\hat{T}$, whose real eigenvalues are $\lambda_{n}$, then the $\mathrm{RH}$ is true. Hence, the zeros $s_{n}$ are consequently given the complex eigenvalues of the operator $1 / 2+i \hat{T}$.

Before constructing the fractal SUSY QM model to prove the RH based on the Hilbert-Polya proposal, let's consider the analogous problem (almost trivial) 
for the $\sinh (s)$ function (the HSRH) described in Section 3. The SUSY QM model involves two isospectral operators $H^{(+)}$and $H^{(-)}$which are defined in terms of the so called SUSY-QM potential $\Phi(x)$. Our ansatz for the SUSY-QM potential associated to the HSRH is given by:

$$
\Phi(x)=\frac{\pi x}{2} .
$$

Note that the SUSY potential is real and it is consistent with the SUSY requirement that $\Phi(x)$ is antisymmetric in $x$ in order to vanish at the origin so that $\Phi^{2}(x)$ is a symmetric function with a minimum at $x=0$ :

$$
\Phi^{2}(x)=\frac{\pi^{2} x^{2}}{4} .
$$

Using such SUSY potential $\Phi$ the following SUSY Schrödinger equation associated with the $\hat{H}^{(+)}$Hamiltonian [42], is:

$$
\left(\frac{\partial}{\partial x}+\Phi\right)\left(-\frac{\partial}{\partial x}+\Phi\right) \psi_{n}^{(+)}(x)=\lambda_{n}^{(+)} \psi_{n}^{(+)}(x),
$$

where we choose the natural units $\hbar=2 m=1$. The isospectral condition of the SUSY-QM model requires that $\lambda_{n}^{(+)}=\lambda_{n}^{(-)}=\lambda_{n}$.

The eigenfunction $\psi_{n}^{(+)}(x)$ associated with the Schrodinger equation for the harmonic oscillator-like potential is the usual Gaussian times a Hermite polynomial and has for corresponding eigenvalues $\lambda_{n}=\hbar \omega(n+1 / 2)$ where the natural frequency is $\omega$.

The potential $V(x)$ of an ordinary QM problem associated with the SUSYQM model is given by (74):

$$
V^{ \pm}(x)=\left[\Phi^{2}(x) \pm \frac{d \Phi(x)}{d x}\right]=\frac{\pi^{2}}{4} x^{2} \pm \frac{\pi}{2} .
$$

The above potentials $V^{ \pm}(x)$ correspond to a harmonic oscillator, whose natural frequency is $\omega=(k / m)^{1 / 2}=\left(\pi^{2} / 2 m\right)^{1 / 2}=\pi$, shifted by an additive postive/negative constant, respectively, and the energy eigenvalues are given by $\lambda_{n}=\pi(n+1)$ and $\pi n$ respectively. In order to have the isospectral condition of SUSY QM $\lambda_{n}^{(+)}=\lambda_{n}^{(-)}=\lambda_{n}$ we must have two different values of $n, n^{\prime}$ such that $n^{\prime}+1=n$. This immediately determines the corresponding eigenfunctions of the two harmonic oscillator partner Hamiltonians.

As we have discussed earlier, the non trivial zeros of the function $\sinh (z)$ are located at $z=0+i n \pi$, for $n=0, \pm 1, \pm 2 \ldots$ which is consistent with the equally spaced eigenvalues of the harmonic oscillator QM problem. This means that it is possible to find an ordinary QM Hamiltonian related to a SUSY-QM model and such that their eigenvalues coincide with the imaginary part of the zeros of $\sinh (z)$. This is the Hilbert-Polya implementation to prove the HSRH in a nontrivial fashion. 
Next, we formulate an inverse eigenvalue problem associated with equation (76), where the $\lambda_{n}$ 's are to be taken as the imaginary parts of the non trivial zeros of our test function $\sinh (s)$. The quantization conditions using the fermionic phase path integral approximation, when aplicable, (the SUSY-QM analog of WKB formula in QM) are based on the CBC formula, the Comtet, Bandrauk and Campbell formula [42]) which reads, after using the natural units $\hbar=2 m$ $=1$, so that all quantities are suitably written in dimensionless variables for simplicity,

$$
I_{n}\left(x_{n}, \lambda_{n} ; a\right) \equiv 4 \int_{0}^{x_{n}} d x\left[\lambda_{n}-\Phi^{2}(x)\right]^{1 / 2}=4 \int_{0}^{x_{n}} d x\left(\lambda_{n}-\frac{\pi^{2} x^{2}}{4}\right)^{1 / 2}=\pi n,
$$

where we take the positive values $n=1,2, \ldots$ and the $\lambda_{n}$ are the imaginary parts of the nontrivial zeros of $\sinh (z)$. The factor of four in equation (78) orginates because one is integrating over a full cycle. The integration between $0, x_{n}$ represents a quarter of a cycle. Due to the fact that $\Phi^{2}(x)$ is an even function of $x$, in order for supersymmetry to be maintained, the left/right turning points obey are symmetrically located: $x_{L}^{(n)}=-x_{R}^{(n)}$ for all orbits, and for each $n=$ $1,2, \ldots$ We define $x_{n}=x_{R}^{(n)}$.

The second set of equations are provided by the location of the turning points of the bound state orbits and which are defined by:

$$
\Phi^{2}\left(x=x_{n}\right)=\lambda_{n}=\pi n ; n=1,2, \ldots
$$

The precise location of the turning points is what is needed in order to evaluate the previous definite integral (the CBC formula) and yield the exact values $\pi n$.

The equations $(77,78,79)$ are the ones we are looking for. The (right) turning points $x_{n}$, are defined in terms of all the $\lambda_{n}$, and the well defined CBC formula is the one which involves the zeros $\lambda_{n}$ associated with the SUSY potential $\Phi(x)$.

Now let us turn to the fractal SUSY QM problem associated to the Riemann Hypothesis. Armitage [43], considered that the RH can be expressed in terms of diffusion processes with an imaginary time. In this way the Hamiltonian of some QM system could be constructed, which in turn implements the Hilbert-Polya's original program.

A numerical exploration of the Hilbert-Polya idea was recently done by $\mathrm{Wu}$ and Sprung [12]. The potential found in [12] has random oscillations around an average value, the average potential allowed them to construct a conventional Hamiltonian whose density of states coincides with the average distribution of the imaginary parts of the Riemann's zeta non trivial zeros. The fluctuations are necessary in order to make the individual eingenvalues fit a set of such zeros within a prescribed error bound. They found that the imaginary parts of the 500 lower lying nontrivial Riemann zeros can be reproduced by a one-dimensional local-potential model, and that a close look at the potential suggests that it has a fractal structure of dimension $D=1.5$. The references [36, 44, 45] deal with fractals (fractal strings) and the Riemann zeta function.

One of us [20], was able to consider a $p$-adic stochastic process having an underlying hidden Parisi-Sourlas supersymmetry, as the effective motion of a 
particle in a potential which can be expanded in terms of an infinite collection of $p$-adic harmonic oscillators with fundamental (Wick-rotated imaginary) frequencies $\omega_{p}=i \ln p$ ( $p$ is a prime) and whose harmonics are $\omega_{p, n}=i \ln p^{n}$. Here, inspired in a work by $\mathrm{Wu}$ and Sprung [12] the $p$-adic harmonic oscillators are substituted by Weierstrass functions. In this way, we propose a way to construct a Hilbert-Polya operator by using (fractal) SUSY-QM arguments.

In SUSY-QM two isospectral operators $H^{(+)}$and $H^{(-)}$are defined in terms of the so called SUSY-QM potential. A SUSY-QM model was proposed in [20] based on the pioneering work of B. Julia [46], where the zeta-function and its fermionic version were related to the partition function of a system of $p$-adic oscillators in thermal equilibrium at a temperature $T$. The fermionic zetafunction has zeros at the same positions of the ordinary Riemann function plus a zero at $1 / 2+0 i$, this zero is associated to the SUSY ground state. See also the reference [14]:

$$
Z_{f}=\frac{\zeta(s)}{\zeta(2 s)}=\sum_{n} \frac{|\mu(n)|}{n^{s}},
$$

where $\mu(n)$ is the Mobius function.

Here we consider a fractal potential, defined by a set unknown phases, to be determined after using the CBC formula, associated with a Weierstrass function, continuous but nowhere differentiable. A fractal SUSY-QM Hamiltonian, using fractional derivatives, can be constructed in principe, whose eigenvalues coincide with the imaginary parts of the nontrivial zeros of the zeta, $\lambda_{n}$. The fractal dimension of the potential is $D=1.5$ and the sought-after phases will be determined by solving the inverse eigenvalue problem via the CBC formula..

Our ansatz for our fractal SUSY-QM potential is based on the Weierstrass fractal function, continuous and nowhere differentiable functions.

$$
W\left(x, \gamma, D, \alpha_{n}\right)=\sum_{n=0}^{\infty} \frac{1-e^{i x \gamma^{n}}}{\gamma^{n(2-D)}} e^{i \alpha_{n}}
$$

$n$ are integers, the powers $\gamma^{n}$ are the corresponding set of frequencies and the $\alpha_{n}$ are the sought-after phases. The expansion (81) is convergent if $1<D<2$ and $\gamma>1$. For these values of the parameters the function $W$ is continuous but nowhere differentiable and has $D$ for fractal dimension $[47,48]$. One could use for the frequencies suitable powers $p^{n}$ of a given prime $p$ number, however, we must study the most general case and have powers $\gamma^{n}$ for all real values of $\gamma>1$.

The aim is to relate the SUSY potential-squared $\Phi^{2}$ to the fractal function $W\left(x, \gamma, D, \alpha_{n}\right)$ defined before. The choice for the $\Phi^{2}(x)$ expression that appears in the fractal version of the CBC formula will be comprised of a smooth part given by the Wu-Sprung potential $V_{W S}(x)$ plus an oscillatory fluctuating Weierstrass part:

$$
\Phi^{2}(x)=V_{W S}(x)+\frac{1}{2}\left[W\left(x, D, \gamma, \alpha_{n}\right)+W\left(-x, D, \gamma, \alpha_{n}\right)+c . c\right]+\phi_{o},
$$


where we have symmetrized the function $W\left(x, D, \gamma, \alpha_{n}\right)$ with respect to the $x$ variables and taken the real part by adding its corresponding complex conjugate (cc). An additive constant $\phi_{o}$ has been included also in order to have a vanishing $\Phi^{2}$ at the origin $x=0$. Supersymmetry requires that the $\Phi^{2}$ is symmetric and vanishes at the origin.

In [12] it was shown that the smooth value of the potential $V_{W S}$ can be obtained as solution of the Abel integral equation. The Wu-Sprung potential $V_{W S}(x)$ is given implicitly as:

$$
x=x(V)=\frac{V_{o}^{1 / 2}}{\pi}\left[(y-1)^{1 / 2} \ln \frac{V_{o}}{2 \pi e^{2}}+y^{1 / 2} \ln \frac{y^{1 / 2}+(y-1)^{1 / 2}}{y^{1 / 2}-(y-1)^{1 / 2}}\right] .
$$

where the rescaled variable is $y=V / V_{o}$, and $V_{o}=3.10073 \pi$.

With the SUSY potential $\Phi$ at hand one may construct the following SUSY Schrödinger equation associated with the $\hat{H}^{(+)}$Hamiltonian [42],

$$
\left(\mathcal{D}^{(\beta)}+\Phi\right)\left(-\mathcal{D}^{(\beta)}+\Phi\right) \psi_{n}^{(+)}(x)=\lambda_{n}^{(+)} \psi_{n}^{(+)}(x),
$$

where we set $\hbar=2 m=1$. The isospectral condition of the SUSY-QM model requires that $\lambda_{n}^{(+)}=\lambda_{n}^{(-)}=\lambda_{n}$. See in [49] an investigation on fractional Laplacians, and in [50] on vector calculus in fractal domains.

The fractal character of the SUSY QM model suggests that equation (84) is actually an stochastic equation. Instead of the usual derivative $d / d x$ we should use the Riemann-Liouville definition of the fractional derivative, as follows,

$$
\mathcal{D}^{(\beta)} F(t)=\frac{1}{\Gamma(1-\beta)} \frac{d}{d t} \int_{-\infty}^{t} \frac{F\left(t^{\prime}\right)}{\left(t-t^{\prime}\right)^{\beta}} d t^{\prime},
$$

where $0<\beta<1$. Similarly, the fractional integral of order $\beta$ is

$$
\mathcal{D}^{(-\beta)} F(t)=\frac{1}{\Gamma(\beta)} \int_{-\infty}^{t} \frac{F\left(t^{\prime}\right)}{\left(t-t^{\prime}\right)^{1-\beta}} d t^{\prime}
$$

where $0<\beta<1$. Notice that the lower limits of integration have been chosen to be $-\infty$. In general these choices may vary.

Several remarks are in order at this point. Firstly, the definition of the fractional derivatives $(85,86)$ is not unique. It depends on the lower limits of the integrals. Rocco and West chose $-\infty$ for the lower limits. Secondly, the fractional derivatives do not obey the Leibniz rule. Therefore: $\mathcal{D}^{\beta}(f g)$ is not equal to $f \mathcal{D}^{\beta} g+g \mathcal{D}^{\beta} f$ which implies that the fractal SUSY QM equation (84) is highly non-trivial because one cannot longer say that the potential can be written naively as $\Phi^{2}+\mathcal{D}^{\beta}(\Phi)$. Thirdly, due to the conditions on the parameter $\beta$ : $0<\beta<1$ in the definitions of fractional derivatives $(85,86)$ to ensure convergence, we cannot take the naive smooth limit $\beta=1$ in the fractal SUSY QM equation (84) and expect to recover the ordinary SUSY QM equation. 
For these three reasons we have not proposed here to naively factorize the ordinary Schrödinger equation in ordear to find the corresponding (smooth) SUSY QM model associated with the smooth Wu-Sprung Schrödinger equation and then perturb it by adding the Weierstrass potential. This would have required also to solve the differential equation: $V_{W S}=\Phi_{\text {smooth }}^{2}+\mathcal{D}^{\beta} \Phi_{\text {smooth }}$, that is highly non-trivial due to the non-analytic nature of the $V_{W S}$ potential. We have done something else which is to write directly the fractal SUSY QM equation (84) in the form given above, instead of factorizing the ordinary Schroedinger equation studied by Wu-Sprung. This latter procedure differs from the former leading to different SUSY QM equations for the three reasons stated earlier.

It is very important to notice this difference and that we cannot take the naive smooth limit $\beta=1$ of (84) in order to arrive at an ordinary SUSY QM equation because the fractal derivatives (anti-derivatives) require the condition on the parameter $0<\beta<1$ to avoid divergences. This explains why we constructed the $\Phi^{2}$ as shown in (82) and then proceeded to use the fractional analog of the CBC formula (87).

With these ingredients we are prepared to manage the inverse eigenvalue problem associated with equation (84), where the $\lambda_{n}$ 's are to be taken as the imaginary parts of the non trivial Riemann zeta zeros. We proceed with our ansatz by showing why $\beta=d=D / 2$ and $D=1.5$. This choice is justified based on the fractal dimension of the Wu-Sprung potential of the order of $D=1.5$ using the first 500 zeros. The reason why $\beta=d=D / 2=3 / 4$ is due to the fact that the two terms which define the fractional (fractal) operator $\mathcal{D}^{(\beta)}+\Phi$ in (84) must have the same fractal dimension. If the fractal $\operatorname{dim}(\Phi)=\mathrm{d}=$ $\operatorname{dim}\left(\mathcal{D}^{(\beta)}\right)=\beta$, according to the properties of $\mathcal{D}^{(\beta)}$ given in [48], if the fractal $\operatorname{dim}(\Phi)=d$, then $\operatorname{dim}\left(\mathcal{D}^{(\beta)} \Phi\right)=\beta+d=2 \beta$. Similarily, for the anti-derivative $\operatorname{dim}\left(\mathcal{D}^{(-\beta)} \Phi\right)=-\beta+d$. Hence, one finally has that the fractal $\operatorname{dim}\left(\Phi^{2}\right)=$ $2 d=\beta+d=2 \beta=D$. From which one infers that $\beta=D / 2=3 / 4$ and it satisfies the required condition for the order $\beta$ of the fractional derivative, $0<\beta<1$.

Therefore, the quantization conditions using the fractal extension of the fermionic phase path integral approximation (the CBC formula) are:

$$
I_{n}\left(x_{n}, \lambda_{n}\right) \equiv 2 \frac{1}{\Gamma(\beta)} \int_{-x_{n}}^{x_{n}} d x^{\prime} \frac{\left[\lambda_{n}-\Phi^{2}\left(x^{\prime}\right)\right]^{1 / 2}}{\left(x_{n}-x^{\prime}\right)^{1-\beta}}=\pi n
$$

where $\beta=D / 2=3 / 4$ and $n=1,2, \ldots$ and $\lambda_{n}$ are the imaginary parts of the nontrivial zeros of zeta. $\Phi^{2}(x, D)$ is an even function of $x$ so the left/right turning points: $x_{L}^{(n)}=-x_{R}^{(n)}$ for all orbits, for each $n=1,2, \ldots$ We define $x_{n}=x_{R}^{(n)}$.

The second set of equations are given by the definition of the turning points of the bound state orbits:

$$
\Phi^{2}\left(x_{n}\right)=\lambda_{n} ; n=1,2, \ldots
$$


So, from the three sets of equations $(82,87,88)$ we get what we are looking for, the relationships among the phases, $\alpha_{n}$, the (right) turning points $x_{n}$, and the imaginary parts of the zeta zeros $\lambda_{n}$.

At this stage is important to say a few words of caution about the normalization of the $\lambda_{n}$ 's. Wu and Sprung studied their QM Schrödinger equation with a fractal potential based on the mean-spacing distribution of the zeros being set to unity. This means that the $\lambda_{n}$ 's to be used in the CBC formula should be properly normalized as follows,

$$
\lambda_{n} \rightarrow \frac{\lambda_{n}}{2 \pi} \log \lambda_{n}
$$

Having said this we can proceed with the determination of the parameter $\gamma>1$ (the frequencies of the Weierstrass function are $\gamma^{n}$ ) will come into play. One still has the freedom to vary such parameter at will. This parameter can be fixed through an optimization procedure. From the formulae above, one has a one-parameter family of phases $\alpha_{n}$ which depend on the values $\lambda_{n}$ as well as the parameter $\gamma>1$. One must go back to the original fractal SUSY QM wave equation to ensure in fact that the SUSY potential $\Phi$ reproduces precisely the original $\lambda_{n}$ for the eigenvalues!. The error terms will depend on the different choices of $\gamma$. The minimization of the error terms should select, in principle, the optimum choice for $\gamma>1$ compatible with the SUSY QM wave equation. It would be intriguing to see if $\gamma=1+\phi=1.618$, the inverse of the golden mean, since the golden mean appears in the the theory of quantum noise related to the $\mathrm{RH}[17]$.

By "fractal" SUSY QM model one means a factorization of a Hamiltonian into two products of operators involving fractional derivatives of irrational order. A model of fractal spin has been studied by da Cruz [21]. Our model must not be confused also with those involving fractional supersymmetries in the string literature.

To conclude, we have a well defined extension of the $\mathrm{CBC}$ formula based on a fractal SUSY QM model, that gives a direct one-to-one correspondence among the imaginary parts of the zeros $\lambda_{n}$ and the phases $\alpha_{n}$. This procedure defines the fractal SUSY QM model which yields the imaginary parts of the zeros of zeta implementing the Hilbert-Polya proposal to prove the Riemann Hypothesis. It is warranted to see if the statistical distribution of these phases $\alpha_{n}$ has any bearing to random matrix theory (the circular unitary random matrix ensemble) and the recent studies of quantum phase-locking, entanglement, Ramanujan sums and cyclotomy studied by [51].

The eigenvalue problem for the $H^{(+)}$Hamiltonian can be reduced to diagonalize an infinite matrix, whose matrix elements can be easily obtained once a convenient basis is found. This matrix involves an infinite set of unknowns in order to have the Riemann's zeros as eigenvalues. A numerical evaluation for each convenient truncation of the matrix is possible. One concludes that the phases of the Weierstrass fractal function appearing in the definition of the $\Phi^{2}(x)$, namely the square of the SUSY-QM potential, Equation (82), $\alpha_{n}$ are 
only approximately found by this method. However this approach has the advantage to give us some clues about the nature and the precise expression of the (square of) SUSY-QM potential. Of course equation (84) could, in principle, be numerically treated following numerical procedures analogous to those used in [12] to give values of the unknown phases $\alpha_{n}$ within prescribed error bounds.

\section{Concluding remarks, $1 / f$ Noise}

In this conclusion we should add the relationship of the Weierstrass function with the Riemann Hypothesis and the $1 / f$ noise. We wish simply to show to why the potential that reproduces the zeta zeros is deeply connected with the theory of $1 / f$ Noise. Here is the simple proof. The amplitudes of the Weierstrass function are given by

$$
\gamma^{n(2-D)}
$$

and the power is proportional to the square of the amplitude and goes as $\gamma^{-2 n(2-D)}$.

Because the frequencies of the Weiersrass function are given by $f_{n}=\gamma^{n}$, this means that the power spectrum falls as $f_{n}^{-2(2-D)}$. Since the fractal dimension of the Weierstrass function (potential) is $D=1.5$, we conclude that one has trully $1 / f$ noise because $f^{-2(2-1.5)}=1 / f$. This concludes the simple proof. See more on $1 / f$ noise in [52].

Thus, the zeta zeros are connected to the $1 / f$ noise va the fractal potential based on a Weierstrass function of fractal dimension $D=1.5$. We believe that the fractal SUSY QM model, once the optimum value for amplitude factor $\gamma$ is known, has a great chance of truly reproducing the zeta zeros, and proving the $\mathrm{RH}$, by simply establishing a one-to-one correspondence among the values of the infinite phases of our Weiertrass function with the zeta zeros.

The equations that yield such correspondence are explictly written in the fractal analog of the CBC formula (87) and after using (88). Berry and Lewis [47] have studed in detail the properties of the Weierstrass-Mandelbrot function, in particular the stochastic behaviour of the phases as well as the behavour for different values of the fractal dimensions and the $\gamma$ factors. See also [53]. The author [54] has discussed the relations among the Riemann hypothesis, Levy flights and fractal random Walks within a probabilistic framework where the Weierstrass function appears.

\section{Acknowledgements}

C. C. is grateful to Tony Smith for many discussions and help, to V. Christianatos for giving us reference [48], to M. Bowers for her hospitality and to Michel Planat and Haret Rosu for many discussions. We thank the Center for Theoretical Studies of Physical Systems, Clark Atlanta University, Atlanta, Georgia, USA, and the Research Committee of the University of Antioquia (CODI), Medellín, Colombia for support. 


\section{References}

[1] B. Riemann. "On the number of prime numbers less than a given quantity". Monatsberichte der Berliner Akademie, November, 1859. Translated by D. R. Wilkins, 1998, available at [14].

[2] A. A. Karatsuba, S. M. Voronin. "The Riemann zeta function". Translated from the Russian by Neal Koblitz. Berlin-New York, Walter de Gruyter Pub., 1992, pages 8-10 in particular.

[3] S. J. Patterson. "An introduction to the theory of the Riemann zeta function". Cambridge, Univ. Press, 1988; H. M. Edwards. "Riemann's Zeta Function". New York, Dover Pub., 2001;

[4] E. C. Titchmarsh. "The theory of the Riemann zeta-function". Oxford, Clarendon Press, 1986.

[5] N. Katz, P. Sarnak. Bull. Am. Math. Soc., 36 (1999) 1.

[6] M. Berry, J. Keating. SIAM Review 41 No. 2 (1999) 236.

[7] A. Connes. Selecta Math. (NS) 5 (1999) 29.

[8] J. Main. Phys. Rep. 316 (1999) 233.

[9] M. A. Selvam has shown that the distribution of vortices and eddies in the cloud formation bears a deep relation to the distribution of the zeros of zeta. See http://www.geocities. com/amselvam/.

[10] H. Montgomery. "Proc. Int. Congress of Mathematics". Vancouver (1974), vol. 1,379 .

[11] M. Planat. Fluctuation and Noise Letters 1 (2001) R65.

[12] H. Wu, D. W. L. Sprung. Phys. Rev. E 48 (1993) 2595. See also

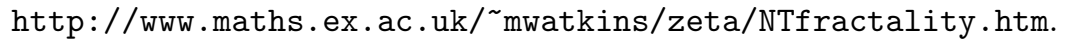

[13] H. Rosu. "Quantum Hamiltonians and Prime numbers". arXiv:quantph/0304139. To appear in Mod. Phys. Let A 18 (2003).

[14] M. Watkins. http://www.maths.ex.ac.uk/mwatkins/zeta/.

[15] M. Pigli. "Adelic integrable systems". arXiv:hep-th/9507031.

[16] L. Fadeev, B. S Pavlov. Sov. Math. Phys. 3 (1975) 522.

[17] M. Planat, S. Perrine. "Arithmetical statistical mechanics: The scene of $1 / f$ noise". Talk at the ninth Van de Ziel symposium, Richmond, Virginia, USA.

[18] L. Brekke, P. Freund. Phys. Reports 233 (1993) 1. 
[19] S. Joffily. "Jost functions, prime numbers and the Riemann zeta". arXiv:math-ph/0303014.

[20] C. Castro. "On p-adic Stochastic Dynamics, Supersymmetry, and the Riemann Conjecture". Jour. Chaos, Solitons and Fractals 15 (2003) 5-24.

[21] W. da Cruz. "A quantum-geometrical description of fracton statistics". arXiv:cond-mat/0212567; W. da Cruz. J. Chaos Sol. and Fract. 17 (2003) 975; W. da Cruz. "The Hausdorff dimension of fractal sets and fractional quantum Hall effect". arXiv:math-ph/0209028.

[22] S. Grynberg, M. N. Piacquadio. "Self-similarity of Farey staircases". arXiv:math-ph/0306024.

[23] J. Franel, E. Landau. Gottinger Nachr (1924) 198.

[24] C. Castro, J. Mahecha. J. Chaos, Sol. and Fract. 13 (2002) 1407.

[25] C. Castro, J. Mahecha. "Final steps towards a proof of the Riemann hypothesis". arXiv:hep-th/0208221.

[26] M. Pitkänen. "A further step in the proof of Riemann hypothesis", arXiv:gen-math/0109072. See also http://www.physics.helsinki.fi/matpitka/.

[27] L. de Branges. J. Funct. Anal. 107 (1992) 122; J. Alcántara-Bode. Int. Eqs. Op. Theory 17 (1993) 151; L. de Branges. J. Funct. Anal. 121 (1994) 117; X.-J. Li. Math. Nachr. 166 (1994) 229; N. Nikolski. Ann. Inst. Fourier (Grenoble) 45 (1995) 143; V.I. Vasyunin. Algebra i Analiz 7 (1995) 118 [Trans. St. Petersburg Math. J. 7 (1996) 405].

[28] E. Elizalde, V. Moretti, S. Zerbini. "On recent strategies proposed for proving the Riemann hypothesis". arXiv:math-ph/0109006.

[29] P. Biane, J. Pitman, M. Yor. "Probability laws related to the Jacobi theta and Riemann zeta functions and Brownian excursions". Technical Report No. 569, U. C. Berkeley, 1999.

[30] C. Castro, A. Granik, J. Mahecha. "On SUSY-QM, fractal strings and steps towards a proof of the Riemann hypothesis". arXiv:hep-th/0107266.

[31] E. Elizalde. "Ten physical applications of spectral zeta functions". Berlin, Springer-Verlag, 1995.

[32] B. E. Heim. "Pullbacks of Eisenstein series, Hecke-Jacobi theory and automorphic L -functions". In "Automorphic forms, automorphic representations, and arithmetic", R. S. Doran, Z. Li Dou, G. T. Gilbert, Editors. AMS Student Mathematical Library, Proceedings of Symposia in Pure Mathematics, Volume 66, (1999).

[33] A. Denjoy. C.R. Acd. Sci. Paris 192 (1931) 656. 
[34] J. Hoppe. Int. Journal Mod. Phys. A 4 (1989) 5235.

[35] G. J. Chaitin. "Thoughts on the Riemann hypothesis". The Mathematical Intelligencer 26 (2004) 4. arXiv:math.HO/0306042.

[36] M. Wolf. Physica A 160 (1989) 24; M. Wolf. Physica A 241 (1997), 493; M. Wolf. Physica A 250 (1998) 335.

[37] C.C. Perelman to Matthew Watkins.

[38] A. Ivic. "On some reasons for doubting the Riemann Hypotesis". arXiv:math.NT/0311162.

[39] A. Odlyzko. "Proc. of the Fourth Int. Conference on Supercomputing". L. P. Kartashev and S. I. Kartshev (eds). Int. Supercomputer Institute (1989), 348 .

[40] J. Ambjorn, J. Jurkiewicz and R. Loll. "Emergence of a $4 D$ world from causal quantum gravity". arXiv:hep-th/0404156.

[41] F. Antonsen. "Zeta-function and star-products". arXiv:quan-ph/9802031.

[42] G. Junker. "Supersymmetric methods in quantum and statistical physics". Springer-Verlag, Berlin, 1996.

[43] J. V. Armitage. "The Riemann hypothesis and the Hamiltonian of a quantum mechanical system", in "Number theory and dynamical systems, M. M. Dodson, J. A. G. Vickers (eds.) (LMS Lecture Notes, series 134, Cambridge University Press), 153.

[44] M. Lapidus, M. van Frankenhuysen. "Fractal geometry and number theory, fractal strings and zeros of the Zeta functions", Birkhauser, 2000.

[45] S. C. Woon. "Riemann zeta function is a fractal". arXiv:chao-dyn/9406003.

[46] B. L. Julia. "Statistical theory of numbers". In Number Theory and Physics, Springer Proceedings in Physics, Vol. 47, J. M. Luck, P. Moussa, M. Waldschmidt (editors). (Springer-Verlag Berlin, Heidelberg 1990) pp. 276.

[47] M. V. Berry, Z. V. Lewis. Proc. Roy. Soc. Lond. A 370 (1980) 459.

[48] A. Rocco, B. J. West. "Fractional calculus and the evolution of fractal phenomena". arXiv:chao-dyn/9810030.

[49] W. Chen, S. Holm. "Fractional Laplacian, Levy stable distribution, and time-space models for linear and nonlinear frequency-dependent lossy media". arXiv:math-ph/0212075.

[50] M. Giona. J. Chaos, Sol. and Fract. 10 (1999) 1349.

[51] M. Planat, H. Rosu. "Cyclotomy and Ramanujan sums in quantum phase locking". To appear in Phys. Lett. A (2003). 
[52] W. Li. "A bibliography on $1 / f$ noise", http://www.nslij-genetics.org/wli/1fnoise.

[53] T. Y. Hu, K. S. Yau. Trans. Am. Math. Soc. 335 (1993) 649; N. Patzschke, M. Zăhle. Stoch. Proc. Appl. 43 (1992) 165; 45 (1993) 61.

[54] M. Schlesinger. Physica A 138 (1986) 310.

[55] H. P. McKean, V. Moll. "Elliptic curves, function theory, geometry and arithmetic". Cambridge, Cambridge University Press, 1999.

[56] B. P. van Zyl, D. A. W. Hutchinson. Phys. Rev. E 67 (2003) 066211.

[57] S.M Gonek. "Three lectures on the Riemann zeta function". arXiv:math.NT/0401126. 


\section{Figures}

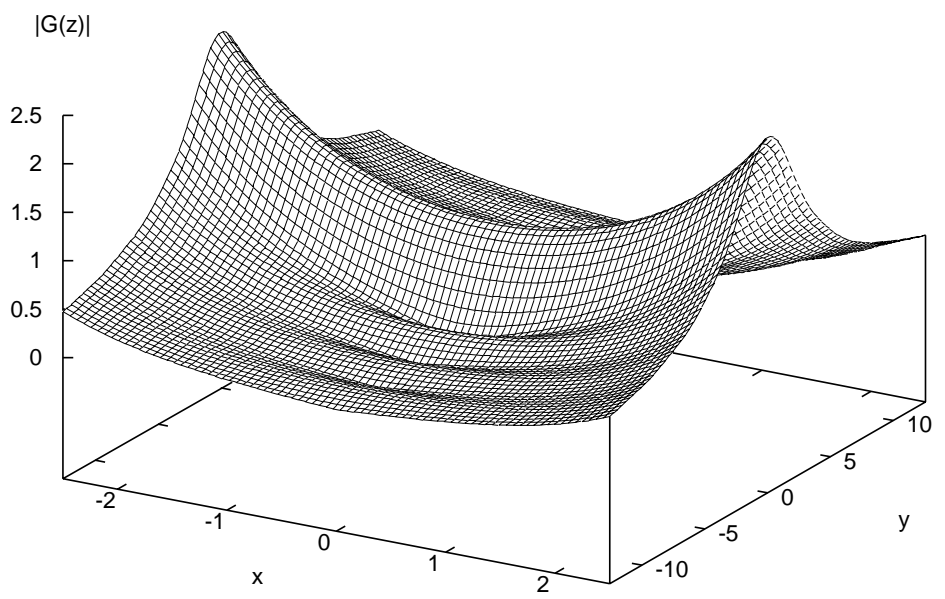

Figure 1: Plot of the absolute value of the function $G(z)=\sinh (z) / z ; z=x+i y$. 


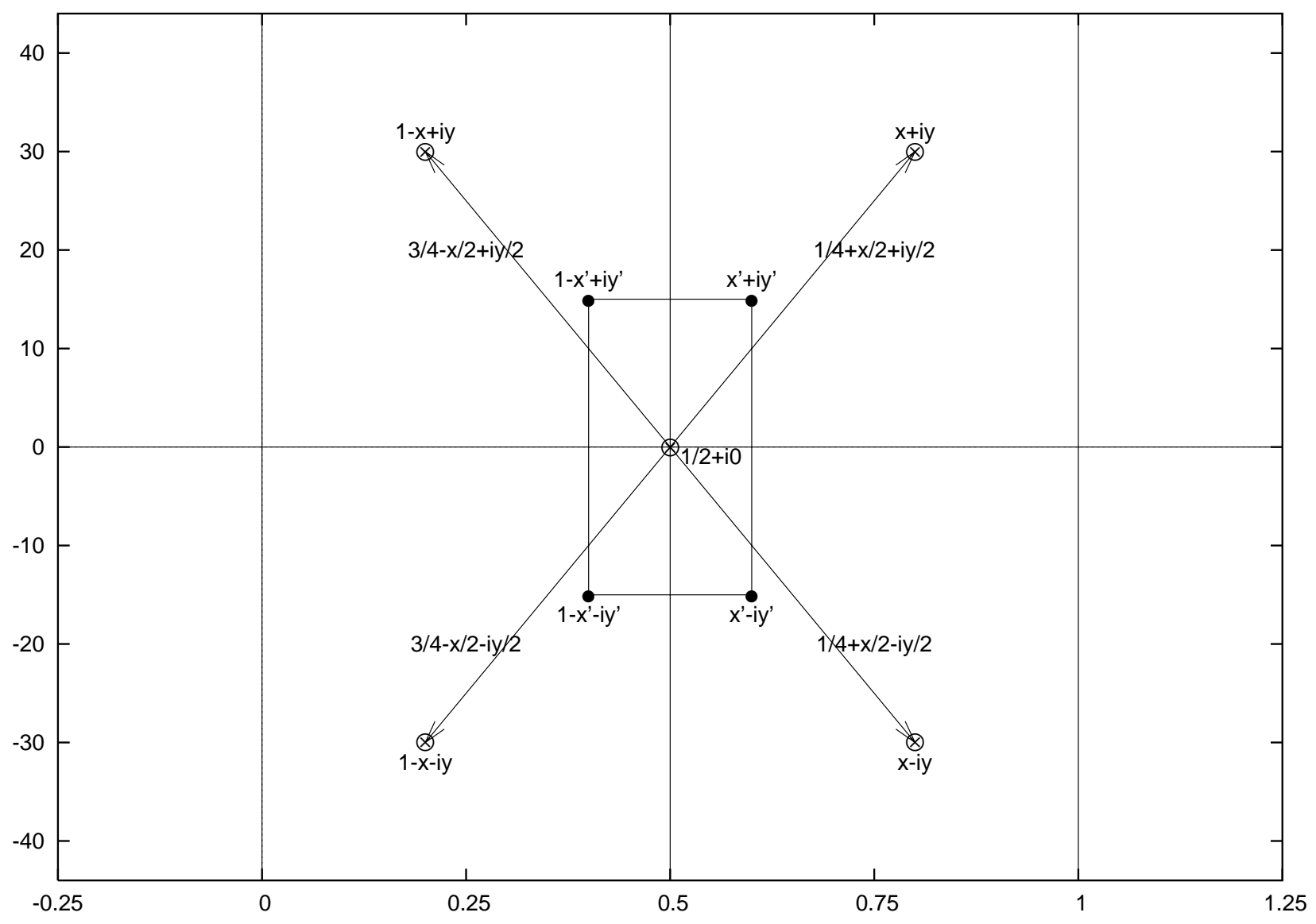

Figure 2: The dots represent generic zeros of the $\zeta$. The crosses represent generic states orthogonal to the reference state $1 / 2+0 i$. The numbers $3 / 4-x / 2-i y / 2$, etc, are the arguments of $Z$ appearing in the orthogonality relations between states orthogonal to the reference state. Due to the functional equation of the Riemann zeta-function, these arguments are just the average values between $1 / 2+0 i$ and those orthogonal states. Here we are referring the particular case $k=1, l=4$. 


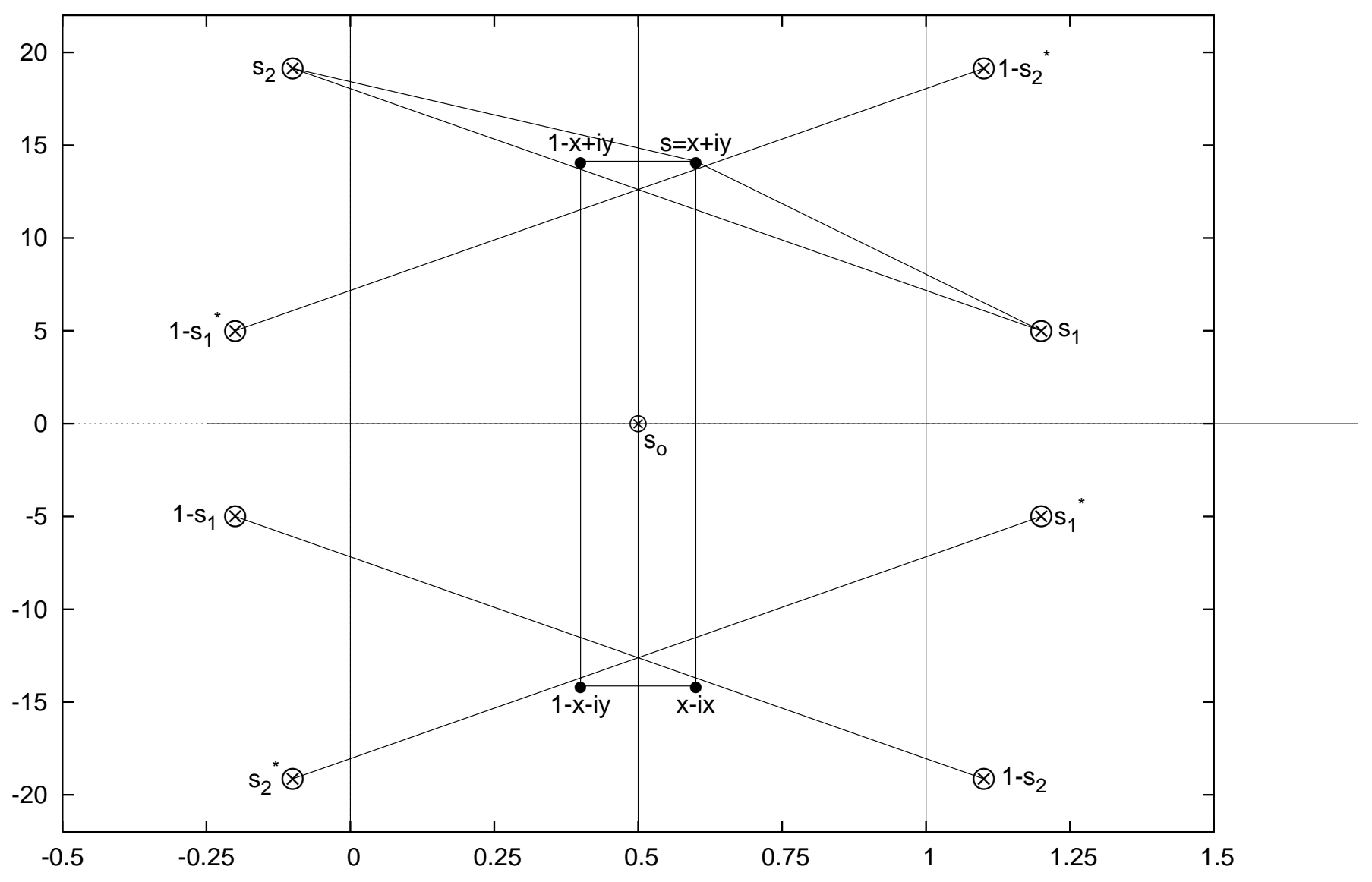

Figure 3: The dots represent generic hypothetic zeros of the $\zeta$. The crosses represent generic states orthogonal between them. Here we are referring the particular case $a=1, b=0$. 
(a)

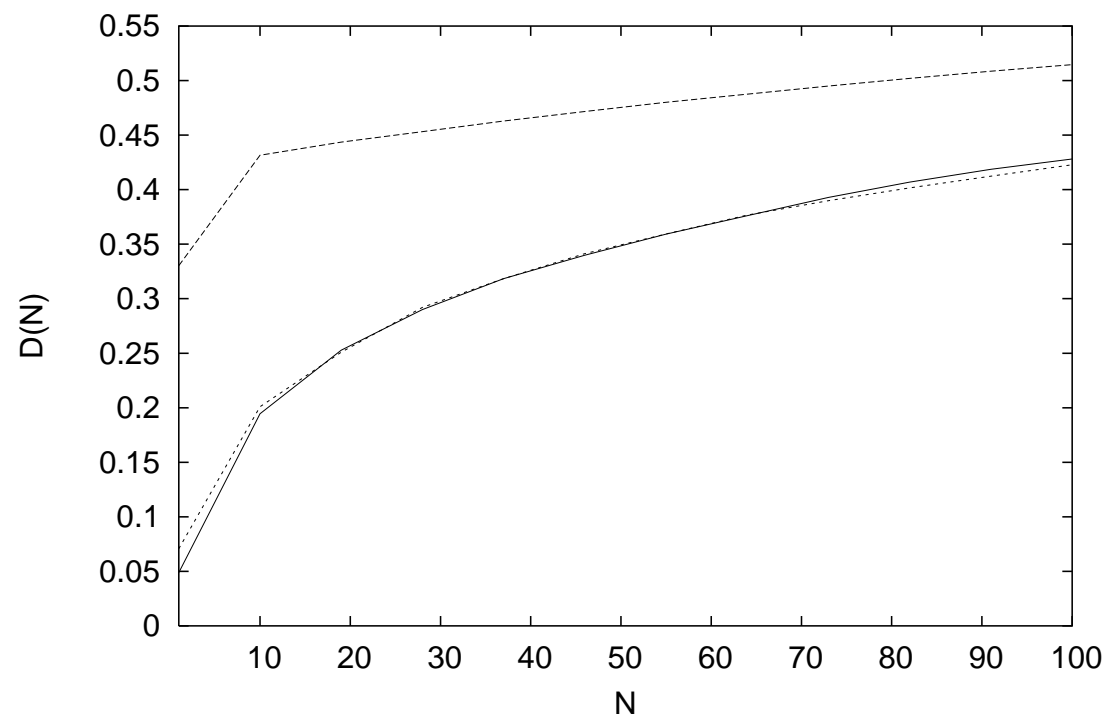

(b)

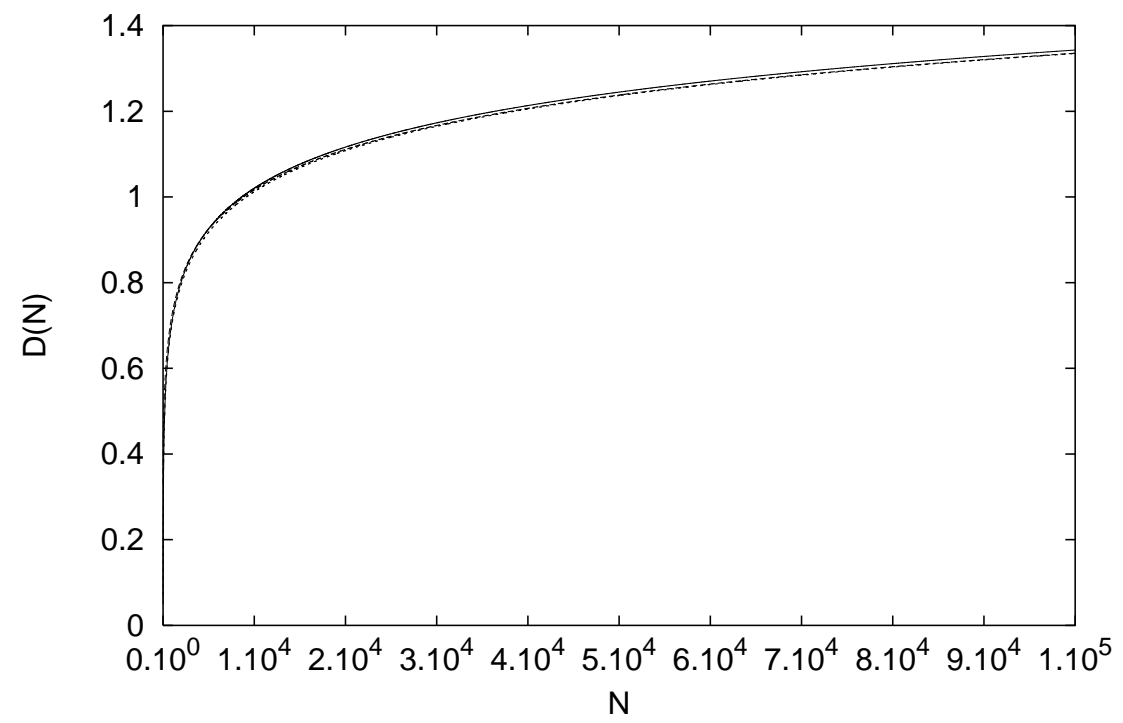

Figure 4: Density of zeros of the Riemann $\zeta$ function in the region $R_{N}$ as a function of $N$. These densities are evaluated by using the formulas (61), (63) and (70). (a) Small $N$ region, calculated with the zeros $s_{n}$ from $n=1$ to $n=100$. (b) Large $N$ region, calculated with the zeros $s_{n}$ from $n=1$ to $n=100000$. We observe that all the three curves converge to each other in the asymptotic region. 
(b)

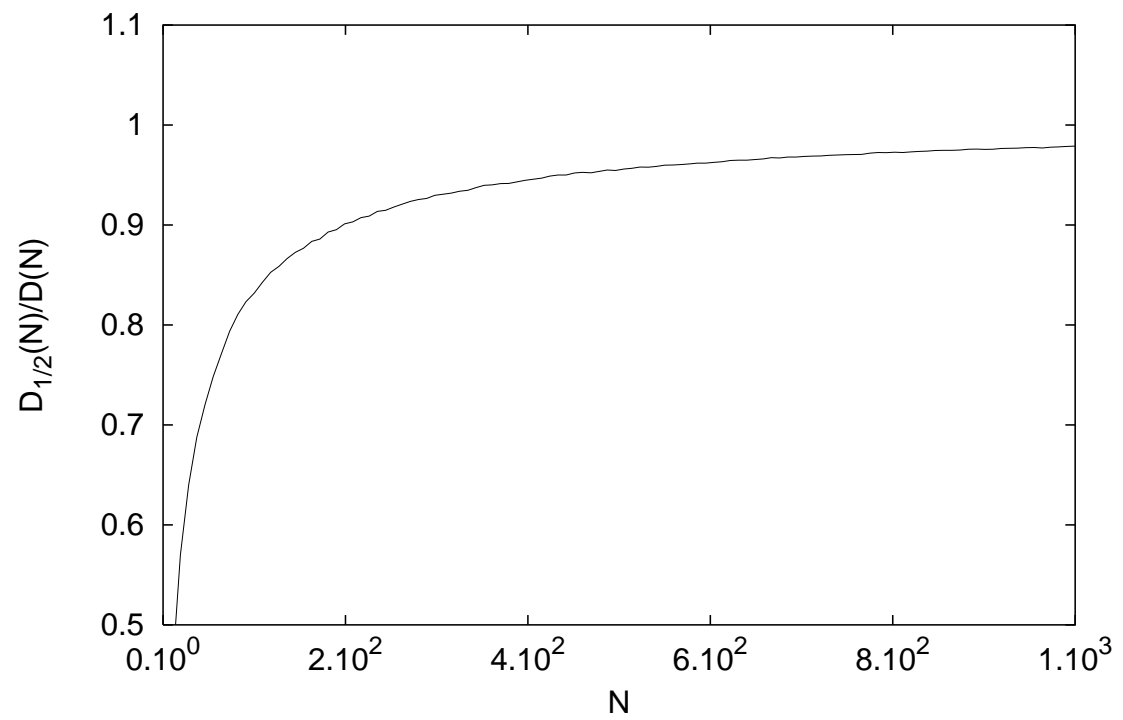

(b)

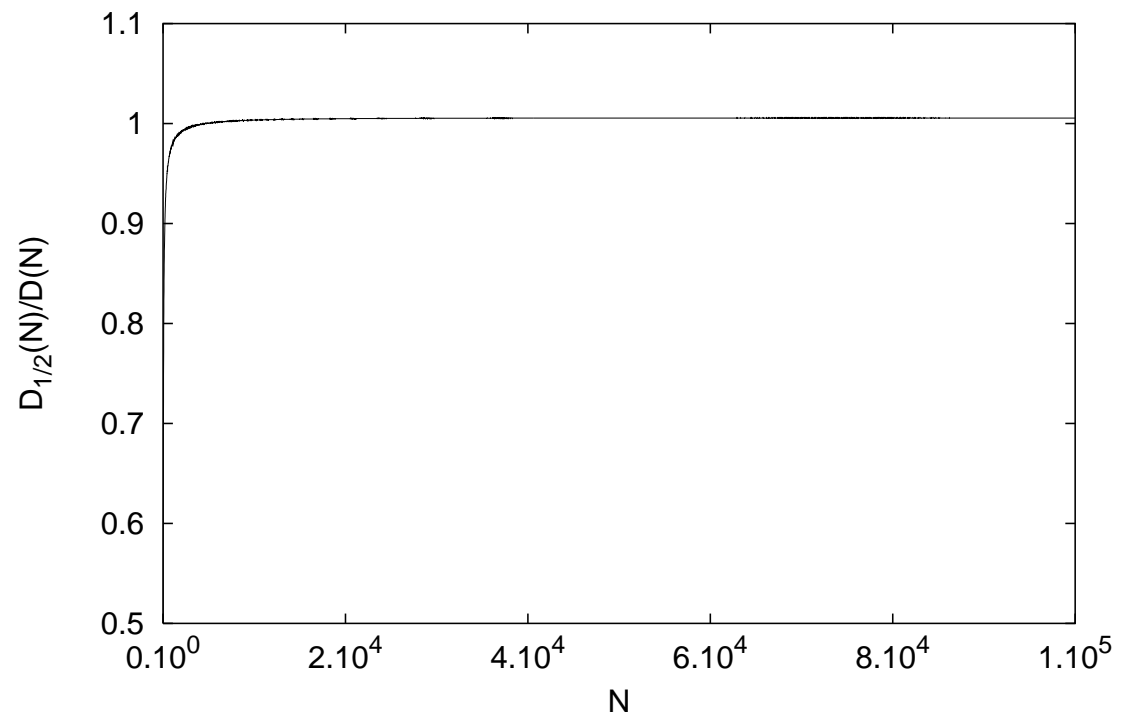

Figure 5: We plot the ratio of the density of zeros of the Riemann $\zeta$ function in the critical line obtained from geometric probability with the density of zeros in the critical strip given explicitly by Equation (73). This density-ratio, in the large $N$ limit, is the sought-after probability to find a zero in the critical line. (a) Small $N$ region. (b) Large $N$ region. Notice that the curves asymptotically approach unity, which is signal that the $\mathrm{RH}$ is true. 
(a)

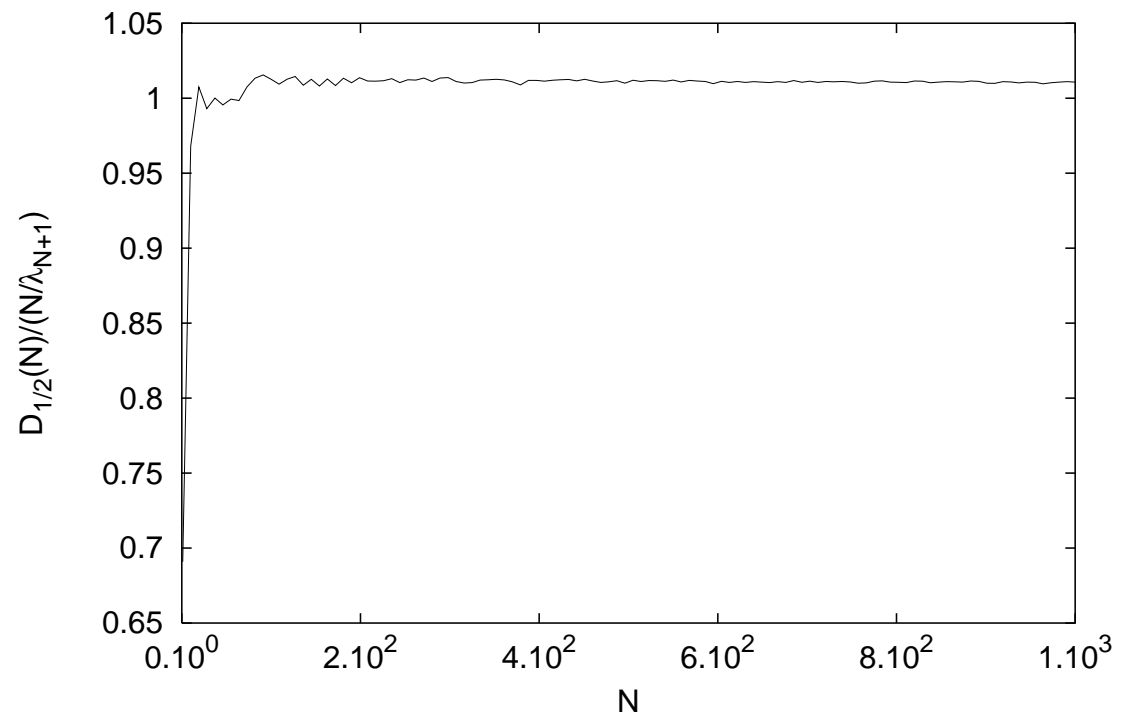

(b)

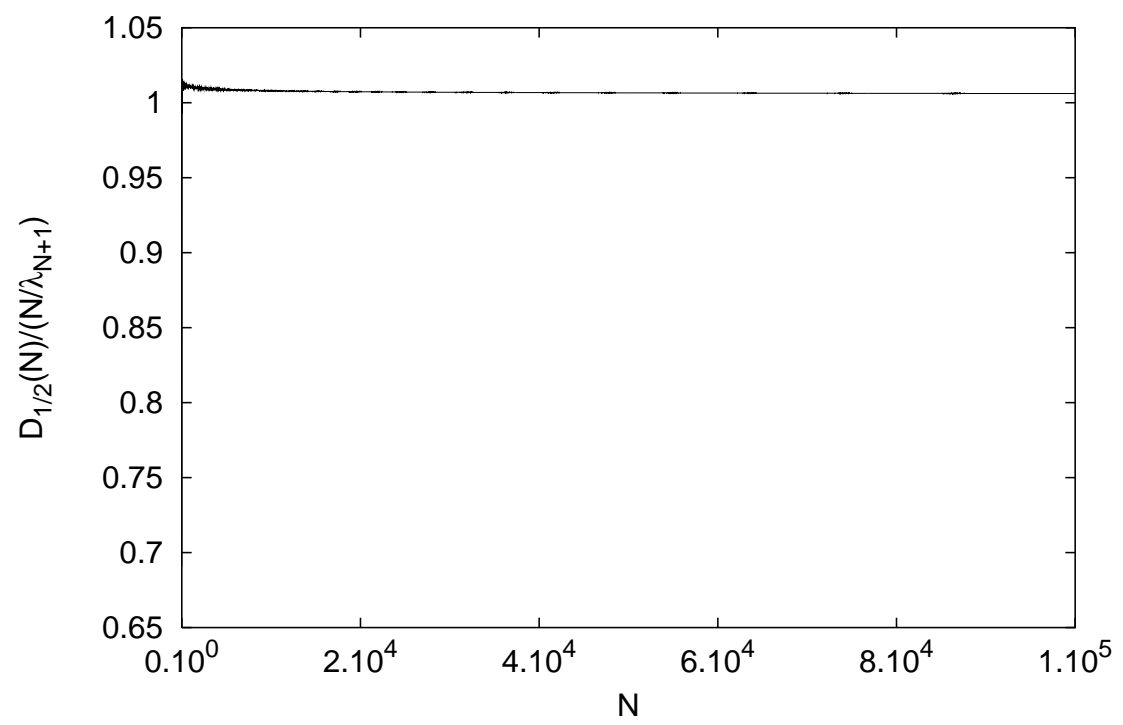

Figure 6: We plot the ratio of the density of zeros of the Riemann $\zeta$ function in the critical line obtained from geometric probability with the standard definiton of a number-density $N_{1 / 2} / \lambda_{N+1}$. See Equations (61), (63) and (70). (a) Small $N$ region. (b) Large $N$ region. We see that the curves asymptotically approach to 1 . 